\title{
CheckV assesses the quality and completeness of metagenome-assembled viral genomes
}

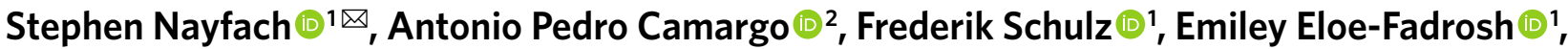 \\ Simon Roux ${ }^{1}$ and Nikos C. Kyrpides ${ }^{1 凶}$
}

\begin{abstract}
Millions of new viral sequences have been identified from metagenomes, but the quality and completeness of these sequences vary considerably. Here we present CheckV, an automated pipeline for identifying closed viral genomes, estimating the completeness of genome fragments and removing flanking host regions from integrated proviruses. CheckV estimates completeness by comparing sequences with a large database of complete viral genomes, including 76,262 identified from a systematic search of publicly available metagenomes, metatranscriptomes and metaviromes. After validation on mock datasets and comparison to existing methods, we applied CheckV to large and diverse collections of metagenome-assembled viral sequences, including IMG/VR and the Global Ocean Virome. This revealed 44,652 high-quality viral genomes (that is, $>90 \%$ complete), although the vast majority of sequences were small fragments, which highlights the challenge of assembling viral genomes from short-read metagenomes. Additionally, we found that removal of host contamination substantially improved the accurate identification of auxiliary metabolic genes and interpretation of viral-encoded functions.
\end{abstract}

$\mathrm{V}$ iruses are the most abundant biological entity on Earth, infect every domain of life and are broadly recognized as key regulators of microbial communities and processes ${ }^{1-4}$. However, it is estimated that only a limited fraction of the viral diversity on Earth can be cultivated and studied under laboratory conditions $^{5}$. For this reason, scientists have turned to metagenomic sequencing to recover and study the genomes of uncultivated viruses $^{6-8}$. Typically, DNA or RNA is extracted from an environmental sample, fragmented and then sequenced, generating millions of short reads that are assembled into contigs. Metagenomic viral contigs are then identified using computational tools and algorithms that use a variety of viral-specific sequence features and signatures ${ }^{9-11}$. In contrast to bacteria and archaea, many viral genomes are sufficiently small that they can be recovered by a single metagenomic contig ${ }^{5-7}$ or a single long read from Nanopore ${ }^{12,13}$ or PacBio technologies ${ }^{14}$. However, metagenome binning may be required for viruses with exceptionally large genomes, such as giant viruses ${ }^{15}$.

Assembly of viruses from metagenomes is challenging ${ }^{16}$ and the completeness of assembled contigs can vary widely, ranging from short fragments to complete or near-complete genomes ${ }^{17}$. Small genome fragments may adversely affect downstream analyses including estimation of viral diversity, host prediction or identification of core genes within viral lineages. Viral contigs can also be derived from integrated proviruses, in which case the viral sequence may be flanked on one or both sides by regions originating from the host genome. Host contamination also adversely affects downstream analyses $^{18}$, especially the estimation of viral genome size, characterization of viral gene content and identification of viral-encoded metabolic genes.

For bacteria and archaea, genome quality can now be readily determined. The most widely adopted method, CheckM, estimates genome completeness and contamination based on the presence and copy number of widely distributed, single-copy marker genes ${ }^{19}$. Because viruses lack widely distributed marker genes, the most commonly used approach with regard to completeness is to apply a uniform length threshold (for example 5 or $10 \mathrm{~kb}$ ) and analyze all viral contigs longer than this ${ }^{5-8}$. However, this 'one-size-fits-all' approach fails to account for the large variability in viral genome size, ranging from $2 \mathrm{~kb}$ in Circoviridae ${ }^{19}$ up to 2.5 megabase pairs $(\mathrm{Mb})$ in Megaviridae ${ }^{20}$, and thus gathers sequences representing a broad range of genome completeness. Complete, circular genomes can be identified from the presence of direct terminal repeat $s^{5-8}$ and sometimes from mapping of paired-end sequencing reads ${ }^{21}$, but tend to be rare. VIBRANT ${ }^{11}$ and viralComplete ${ }^{22}$ are two recently published tools utilized to address these problems: VIBRANT categorizes sequences into quality tiers based on circularity and the presence of viral hallmark proteins, as well as nucleotide replication proteins, while viralComplete estimates completeness based on affiliation to known viruses from NCBI RefSeq.

With regard to host contamination on proviruses, existing approaches either remove viral contigs containing a high fraction of microbial genes ${ }^{5}$ or predict host-virus boundaries ${ }^{10,11,23,24}$. The former approach allows for a small number of microbial genes while the latter may fail to identify a host region or misidentify the true boundary. Other approaches detect viral signatures, but do not explicitly account for the presence of microbial regions. With the diversity of available viral prediction pipelines and protocols, there is a need for a standalone tool to ensure that viral contigs do not contain contamination, and to remove it when present.

Here we present CheckV, a tool used for automatic estimation of genome completeness and host contamination for single-contig viral genomes. Based on benchmarking, we show that CheckV is computationally efficient and considerably more accurate than existing approaches. By collecting an extended database of complete viral genomes from both isolates and environmental samples, CheckV was able to estimate completeness for the vast majority of viral contigs in the IMG/VR database, illustrating its broad applicability to newly assembled genomes across viral taxa from Earth's biomes. In addition, CheckV compares gene content between adjacent windows along each sequence to identify putative host

'US Department of Energy Joint Genome Institute, Lawrence Berkeley National Laboratory, Berkeley, CA, USA. ${ }^{2}$ Department of Genetics, Evolution, Microbiology and Immunology, Institute of Biology, University of Campinas, Campinas, Brazil. e-mail: snayfach@lbl.gov; nckyrpides@lbl.gov 

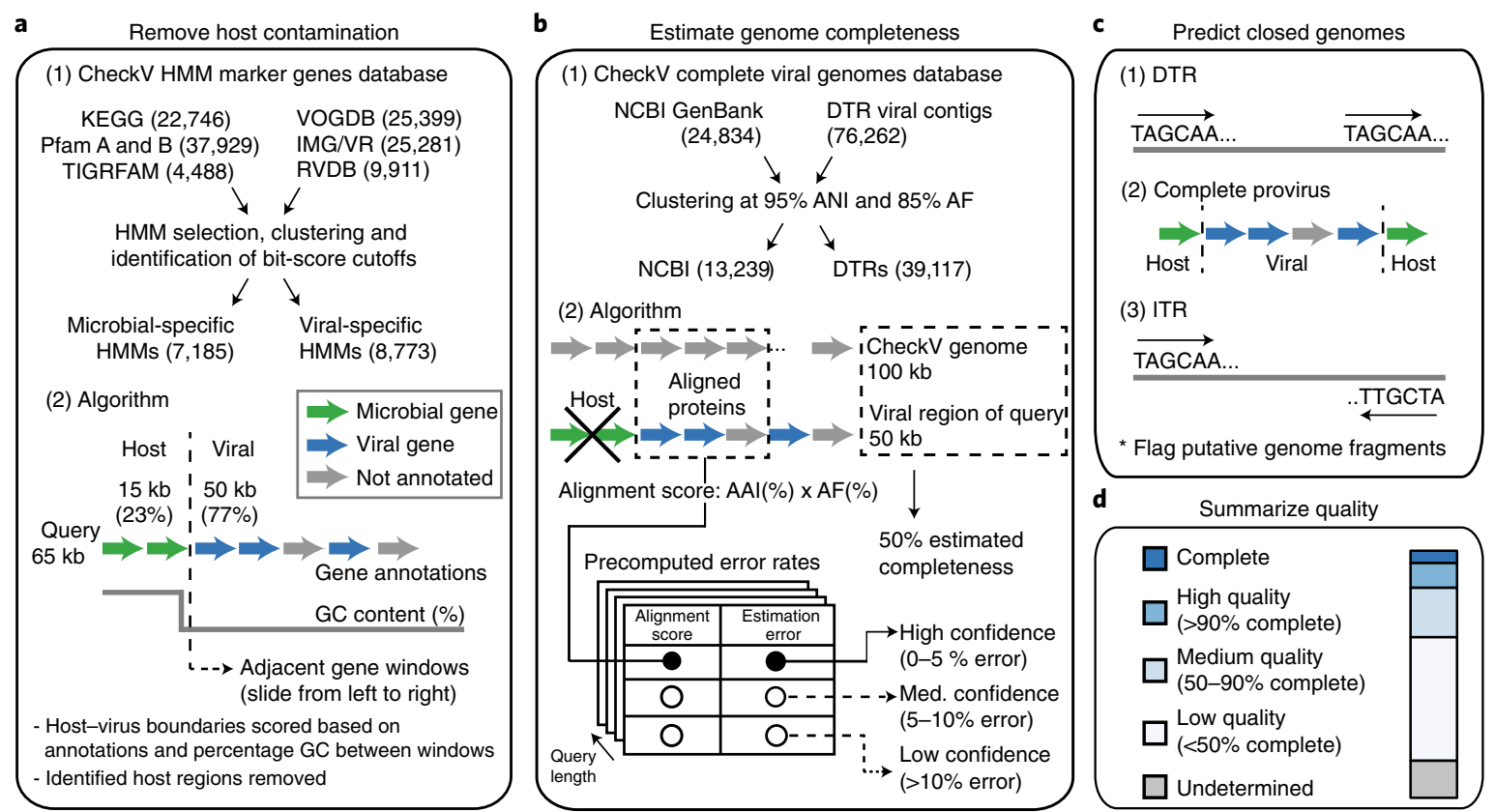

Fig. 1 | A framework for assessing the quality of single-contig viral genomes. a-c, Check $V$ estimates the quality of viral genomes from metagenomes in four main steps. a, First, CheckV identifies and removes nonviral regions on proviruses using an algorithm that leverages gene annotations and GC content. b, CheckV estimates genome completeness based on comparison with a large database of complete viral genomes derived from NCBI GenBank and environmental samples, and reports a confidence level for the estimate. c, Closed genomes are identified based on either direct terminal repeats, flanking host regions on proviruses or inverted terminal repeats. When possible, these predictions are cross-referenced with the completeness estimates obtained in $\mathbf{b}$. Unsupported predictions are flagged as genome fragments. $\mathbf{d}$, Sequences are then assigned to one of five different quality tiers based on their estimated completeness.

contamination stemming from the assembly of integrated proviruses. Application to the IMG/VR database revealed that this type of contamination is rare but could easily lead to misinterpretation of viral genome size and viral-encoded metabolic genes.

\section{Results}

A framework for assessing the quality of single-contig viral genomes. CheckV is a fully automated, command-line tool used for assessing the quality of single-contig viral genomes. It is organized into three modules which identify and remove host contamination on proviruses (Fig. 1a), estimate completeness for genome fragments (Fig. 1b) and predict closed genomes based on terminal repeats and flanking host regions (Fig. 1c). Based on these results, the program classifies each sequence into one of five quality tiers (Fig. 1d) - complete, high quality ( $>90 \%$ completeness), medium quality (50-90\% completeness), low quality (0-50\% completeness) or undetermined quality (no completeness estimate available)-which are consistent with and expand upon the Minimum Information about an Uncultivated Virus Genome (MIUViG) standards ${ }^{17}$. Because host contamination is easily removed, it is not factored into these quality tiers. At present, CheckV is not optimally suited for multi-contig viral genomes (for example, those identified from metagenome binning), which may contain contamination from other viruses or cellular organisms.

In the first step, CheckV identifies and removes nonviral regions on the edges of contigs, which can occur on assembled proviruses (Fig. 1a and Methods). Genes are first annotated as either viral or microbial (that is, from bacteria or archaea) based on comparison to a large database of 15,958 profile hidden Markov models (HMMs) (Extended Data Fig. 1 and Supplementary Table 1). We selected these HMMs from seven reference databases using three main criteria: high specificity to either viral or microbial proteins, commonly occurring in either viral or microbial genomes and nonredundant compared to other HMMs. Starting at the 5' edge of the contig, CheckV compares the genes between a pair of adjacent windows to identify host-virus boundaries characterized by a large difference in gene content (that is, microbial versus viral) and/or nucleotide composition. We optimized this approach to detect flanking host regions sensitively and specifically, even those containing just a few genes.

In the second step, CheckV estimates the expected genome length of contigs based on the average amino acid identity (AAI) to a database of complete viral genomes from NCBI and environmental samples (Fig. 1b and Methods). The expected genome length is then used to estimate completeness as a simple ratio of lengths, similar to previous approaches ${ }^{22,25,26}$. In contrast to bacteria and archaea, genome length is relatively invariant among related viruses (particularly at the family or genus $\operatorname{rank}^{17}$ ), which may be a result of conserved structural features or capsid size. For example, CheckV reference genomes differed by only $12.5 \%$ in length on average (interquartile range (IQR) $=4.2-16.7 \%$ ) for viruses displaying just 30-40\% AAI (Extended Data Fig. 2).

Highly novel viruses may not display sufficient similarity to CheckV reference genomes for accurate estimation of completeness. To address this, CheckV reports a confidence level for each AAI-based estimate according to the expected relative unsigned error rate: high confidence ( $0-5 \%$ error), medium confidence (5-10\% error) or low confidence $(>10 \%$ error). When AAI-based completeness cannot be accurately determined ( $>10 \%$ error), CheckV implements a secondary approach in which the contig length is compared with that of reference genomes that are annotated by the same viral HMMs. Using this information, CheckV reports the range of completeness values corresponding to the fifth and 95th percentiles from the distribution of reference genome lengths (for example, 35-60\% completeness). Compared to the AAI approach, the HMM approach can be more sensitive but is not 
as precise since it reports a range rather than a specific point estimate. Lastly, we designed CheckV so that its database can be readily updated to incorporate novel viral genomes as they are released in public databases (for example, NCBI GenBank and IMG/VR) or discovered from new metagenomics studies.

In the final step, CheckV predicts closed genomes based on three established approaches: direct terminal repeats (DTRs), inverted terminal repeats (ITRs) and integrated proviruses ${ }^{17}$. DTRs and ITRs are identified based on a repeated sequence of at least 20 base pairs (bp) at the start and end of the contig. While DTRs can play a role in genome integration ${ }^{27}$, they often result from assembly of short reads from a circular genome ${ }^{28}$ or a linear genome replicated by a mechanism involving a concatemer intermediary ${ }^{29}$. Pairwise alignment of DTR contigs from closely related viruses can be used to determine whether genomes have been circularly permuted ${ }^{12}$. Inverted terminal repeats are a hallmark of transposons ${ }^{30}$ but have also been observed in complete viral genomes ${ }^{31}$ and phages ${ }^{32}$. Lastly, complete proviruses are identified by a viral region flanked by host DNA on both sides and are commonly found in microbial (meta)genomes ${ }^{10,11,23,24}$. While these are well-established approaches, false positives have also been observed ${ }^{33}$ and so, to mitigate this, CheckV reports a confidence level for putative complete genomes based on the estimated completeness from the AAI- or HMM-based approaches: high confidence ( $\geq 90 \%$ completeness), medium confidence (80-90\% completeness) or low confidence ( $<80 \%$ completeness).

An expanded database of complete viral genomes from Earth's biomes. We initially formed the CheckV genome database using 24,834 viral genomes from NCBI GenBank ${ }^{34}$ (Supplementary Table 2). However, uncultivated identified viruses commonly display little to no similarity to reference databases 5 . To mitigate this issue and expand the diversity of the database, we used CheckV to perform a systematic search for metagenomic viral contigs with DTRs (DTR contigs) from $>14.4$ billion contigs ( 9.7 terabase pairs) derived from publicly available and environmentally diverse metagenomes, metatranscriptomes and metaviromes downloaded from the following: IMG/ $\mathrm{M}^{35}, \mathrm{MGnify}^{36}$ and recently published studies of the human microbiome ${ }^{37-39}$ and ocean virome ${ }^{6}$ (Fig. 2 and Methods). We exclusively used DTRs to identify complete genomes because this is the most well-established approach ${ }^{5-8}$.

Using this approach, we identified 76,262 DTR contigs after carefully filtering out potential false positives and verifying completeness (Extended Data Fig. 3 and Supplementary Table 3). These were subsequently dereplicated to 39,117 sequences at $95 \%$ average nucleotide identity (ANI) over $85 \%$ of the length of both sequences (Supplementary Table 4). DTR contigs were found in diverse environments including the human gut (35.8\%), marine (19.7\%), freshwater $(9.7 \%)$ and soils $(7.0 \%)$ and were derived from major clades of DNA viruses, including Caudovirales (69.1\%), Microviridae (11.4\%) and CRESS viruses (2.3\%) (Fig. 2a,b). DTR contigs were also identified for retroviruses (Retrovirales, $n=1,698$ ) and RNA viruses (Riboviria, $n=83$ ), which were further confirmed through identification of marker genes (for example, $R d R p$ ) and association with known viral families (Supplementary Information).

Next, we compared the 76,262 DTR contigs to the 24,834 GenBank references and dereplicated all sequences again at 95\% ANI, resulting in 52,141 clusters. Overall, the addition of DTR contigs resulted in a $294 \%$ increase in the number of viral clusters, which was particularly pronounced for the order Caudovirales (611\% increase) (Fig. 2b). In contrast, GenBank genomes had improved representation of other viral clades, including RNA viruses from the Riboviria realm (Supplementary Table 5). For most viral clades, the sizes of DTR contigs and GenBank genomes were consistent, indicating no systematic artifacts in our data (Fig. 2b). One interesting exception was for segmented RNA viruses (Riboviria and Retrovirales), in which the DTR contigs tended to be smaller than the GenBank references, suggesting that they either represent a single genome segment or cover only a subset of the diversity within these large groups.

Accurate estimation of genome completeness for novel viruses. Having developed the CheckV pipeline and databases, we next benchmarked its accuracy. To evaluate genome completeness, we generated a mock dataset containing fragments from 2,000 uncultivated, complete viral genomes derived from IMG/VR (Supplementary Table 6). We first estimated completeness using the AAI-based approach after removal of all closely related sequences from the CheckV database ( $>95 \%$ AAI). This revealed a strong correlation with expected values (Pearson's $r=0.941$ ), low overall error $(2.5 \%$ median unsigned error (MUE), IQR $=0.91-5.8 \%)$ and estimates for $97 \%$ of sequences (Fig. 3a). As expected, accuracy varied according to the confidence level, with best performance for high- and medium-confidence estimates (Pearson's $r=0.986$ and 0.945 , respectively). Next we applied the HMM-based approach, which yielded estimated completeness for $89.4 \%$ of fragments and $94.5 \%>5 \mathrm{~kb}$. While HMM-based estimates were considerably less accurate (Pearson's $r=0.871$ ), the lower bound of the estimated range was consistently below the expected value (96.6\% of the time; Fig. 3b). This indicates that the HMM-based approach can be useful for accurately obtaining a conservative lower bound on genome completeness, particularly for novel viruses that display low AAI to CheckV reference genomes.

For comparison, we applied viralComplete ${ }^{22}$ and VIBRANT ${ }^{11}$ to the mock dataset (Fig. 3c,d and Supplementary Table 6). viralComplete estimates completeness based on affiliation to viruses from the NCBI RefSeq database, while VIBRANT classifies sequences into quality tiers based on gene content and the presence of DTRs. VIBRANT's quality tiers were only weakly correlated with true completeness (Pearson's $r=0.466$ ), with a majority of near-complete genomes ( $>90 \%$ complete) classified as either low or medium quality $(183 / 227,80.6 \%)$. viralComplete showed much better performance (Pearson's $r=0.703$ ), but still displayed high error for a considerable number of sequences $(\mathrm{MUE}=8.71 \%, \mathrm{IQR}=3.13-21.76 \%)$.

Based on the presence of clade-specific marker genes, we determined that most sequences from the mock dataset belonged to the Caudovirales order of double-stranded DNA phages. To evaluate performance for other types of viruses, we applied CheckV to viral genome fragments from NCBI GenBank after removal of closely related genomes from the CheckV database (Supplementary Table 7). Using the AAI-based approach (excluding low-confidence estimates), completeness was accurately estimated overall (MUE $=1.33 \%, \mathrm{IQR}=0.45-3.57 \%)$ including for viruses from different Baltimore classes, those infecting various hosts and those from different families (Fig. $3 \mathrm{e}-\mathrm{g}$ ). A few viral groups were problematic, including single-stranded DNA plant viruses from the family Geminiviridae (MUE $=22.2 \%$ ), where CheckV did a poor job of distinguishing between monopartite and segmented genomes, and the Asfarviridae family (170-190kb), where CheckV identified the giant viruses Pacmanvirus (395.4 kb) and Kaumoebavirus (350.7 kb) as nearest relatives in the database.

While CheckV is not ideally suited for viral bins, we evaluated its performance on a recent dataset of 2,074 giant virus metagenome-assembled genomes (GVMAGs) ${ }^{15}$ in which Schulz et al. estimated genome completeness based on 20 low-copy number nucleocytoplasmic virus orthologous groups (NCVOGs) ${ }^{15}$ (Extended Data Fig. 4a). Using the AAI-based approach, CheckV estimated completeness for 6.6, 75.1 and $18.3 \%$ of GVMAGs at the high-, medium- and low-confidence levels, respectively. Overall, CheckV completeness estimates were correlated with those from the NCVOG approach (Pearson's $r=0.451$ ), but particularly for high-confidence CheckV estimates (Pearson's $r=0.705$ ). Similar results were observed based on an analysis of genome fragments from nucleocytoplasmic large DNA virus isolates ${ }^{40}$ (Extended Data 
a

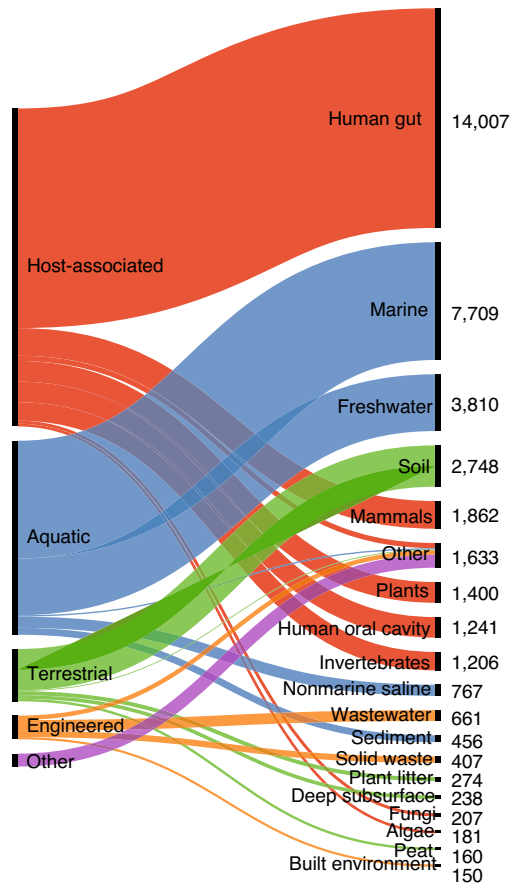

b

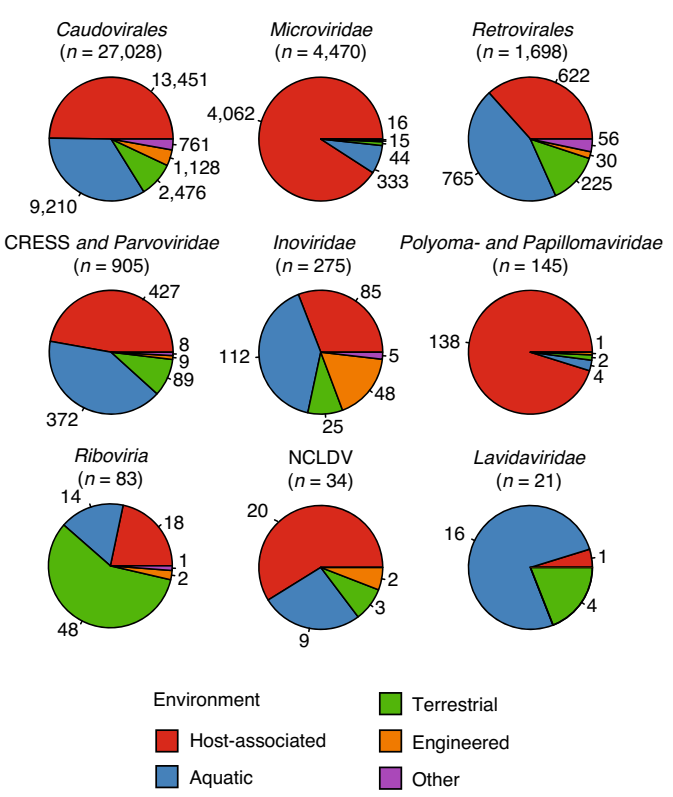

c

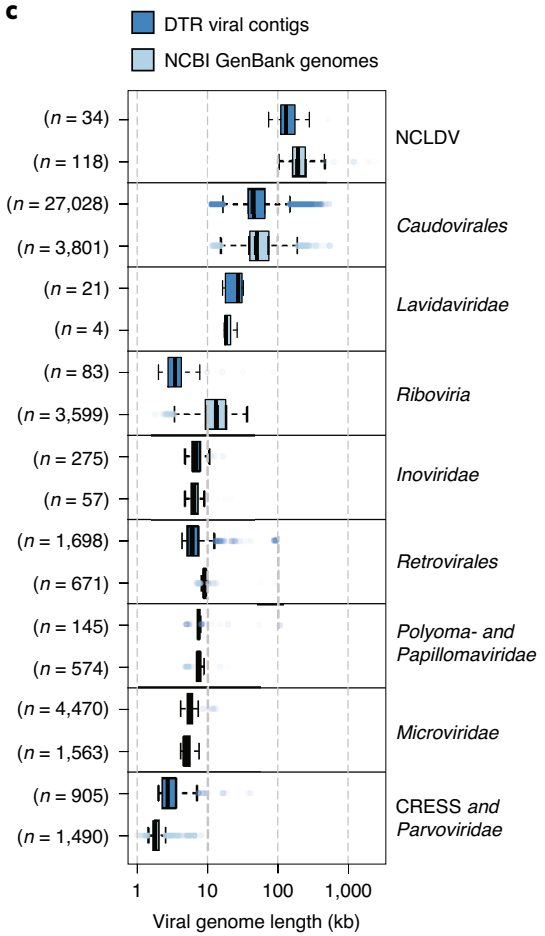

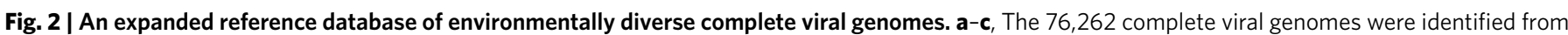
publicly available metagenomes, metatranscriptomes and viromes based on the presence of a DTR and were clustered into 39,117 nonredundant genomes at $95 \%$ ANI. a, The nonredundant genomes are derived from diverse human-associated and environmental habitats. Habitats are based on the Genomes

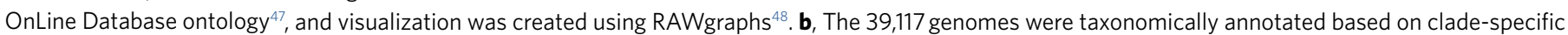

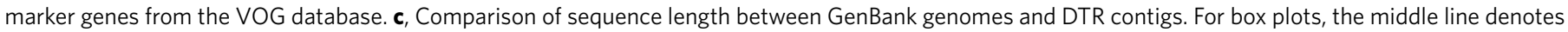
the median, the box denotes IQR and the whiskers denote 1.5×IQR. NCLDV, nucleocytoplasmic large DNA virus.

Fig. 4b). These correlations imply that CheckV gave broadly similar results compared to those of Schulz et al. ${ }^{15}$, and may be suitable for evaluation of the completeness of certain metagenome-assembled giant virus genomes.

Accurate identification of host regions on proviruses. Next, we evaluated CheckV's accuracy in detecting host contamination on provirus sequences (Supplementary Table 8 ). To generate mock proviruses, we identified 382 bacteriophages from NCBI and paired them with their bacterial and archaeal hosts from the Genome Taxonomy Database (GTDB) ${ }^{41}$. After inserting each phage at a random location in its host genome, we extracted genomic fragments varying in both length $(5-50 \mathrm{~kb})$ and amount of flanking host sequence $(10-50 \%)$. Overall, CheckV detected the presence of host regions on $69.0 \%$ of mock proviruses and $88.3 \%$ for contigs $\geq 20 \mathrm{~kb}$ (Fig. 4a). The length of host regions was also accurately estimated (Fig. 4b), with a median unsigned error of only $0.6 \%$ (IQR $=0.16-$ $2.2 \%)$. As a negative control, we applied CheckV to genomic fragments that were entirely viral (that is, no flanking host region). Only $0.80 \%$ of these sequences were classified as proviruses, indicating that $\mathrm{CheckV}$ has a low provirus false-positive rate (Fig. 4c). Similar results were observed when we applied CheckV to complete uncultivated viral genomes from IMG/VR (Fig. 4d and Supplementary Table 9).

For comparison, we evaluated four other tools to identify hostprovirus boundaries, including VIBRANT ${ }^{11}$, VirSorter ${ }^{10}$, PhiSpy $^{23}$ and Phigaro ${ }^{24}$. Compared to these four tools, CheckV displayed consistently higher sensitivity but, in particular, when fragments were short or host contamination was low (Fig. 4a). For example, VirSorter detected only $1.2 \%$ of proviruses with $10 \%$ contamination compared to $57.2 \%$ with CheckV. This implies that microbial genes at the edges of proviral contigs may be overlooked by existing tools and interpreted as viral encoded. In contrast, other tools identified host-virus boundaries on entirely viral sequences (Fig. 4c,d). For example, PhiSpy predicted nonviral regions on $22.9 \%$ of entirely viral fragments that covered $26.3 \%$ of the length of these sequences on average. This implies that truly viral regions may be discarded with existing tools and that sequences may be inadvertently classified as integrated proviruses. Apart from CheckV, VIBRANT performed optimally at identification of flanking host regions on proviruses.

Finally, we compared the computational efficiency of CheckV to existing tools. Using 16 CPUs (Intel Xeon E5-2698 v3 processors), the full CheckV pipeline was 1.6- to 11.6-fold faster than the other programs when applied to the mock dataset and required $\sim 2 \mathrm{~GB}$ of memory. Using a single CPU, CheckV was still faster than VirSorter and VIBRANT but slower compared to viralComplete, PhiSpy and Phigaro (Supplementary Table 10).

Using CheckV to identify high-quality genomes from metagenomes and viromes. To illustrate the type of results obtained with CheckV and its ability to scale to large datasets, we applied it to 735,106 viral contigs from the IMG/VR 2.0 database $^{25}$ (Supplementary Table 11). IMG/VR contigs were previously identified from short-read metagenomic assemblies using the Earth's Virome Protocol ${ }^{5}$ and a minimum contig length cutoff of $5 \mathrm{~kb}$. The original samples were derived from many studies, the majority of which did not use size filtration to enrich for extracellular viral particles. Because of the sample characteristics and detection approach, this dataset is mostly composed of environmental dsDNA phages from the Caudovirales order and contains sequences from both lysogenic and lytic viruses. 


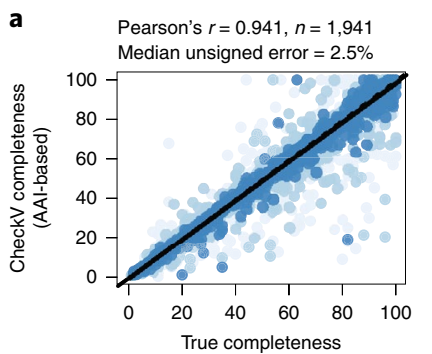

O High confidence ( $r=0.986, n=646)$ O Medium confidence $(r=0.945, n=801)$ O Low confidence $(r=0.877, n=494)$

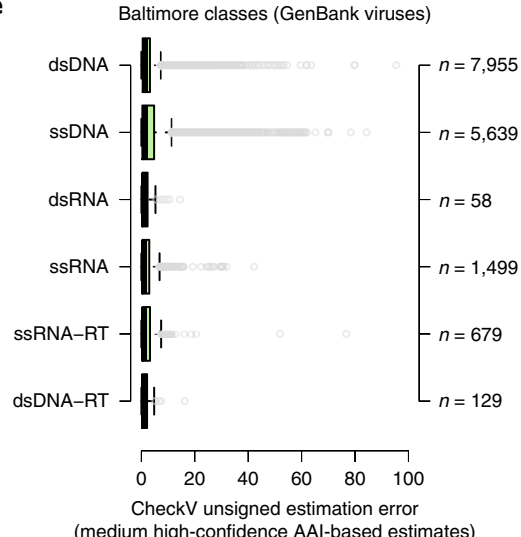

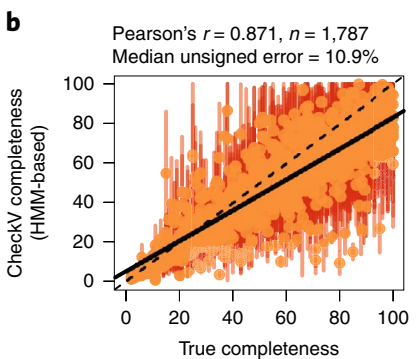

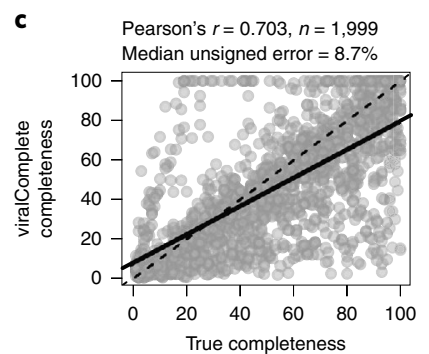

d

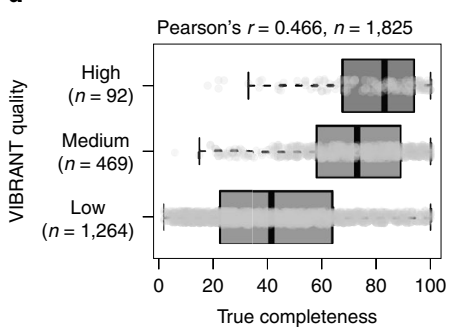

- $90 \%$ confidence interva

○ Midpoint of interval

f

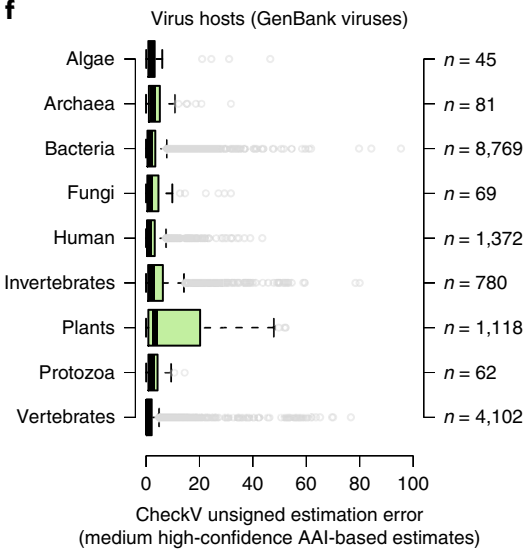

g

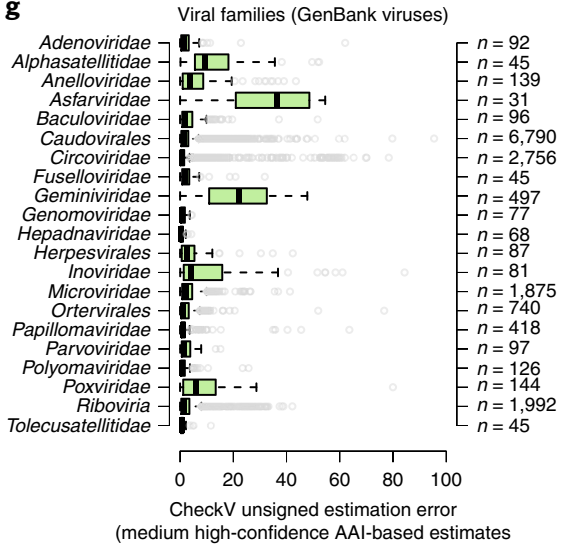

Fig. 3 | Benchmarking completeness estimation for CheckV and existing tools. a-d, Benchmarking completeness for a mock dataset containing fragments from 2,000 complete genomes derived from IMG/VR. Dashed lines represent the line of equality while solid lines indicate best fit. Completeness estimates above $100 \%$ were set to $100 \%$. a, CheckV-estimated completeness using the AAl-based approach. Point color depth indicates estimation confidence level. b, CheckV-estimated completeness using the HMM-based approach. Red vertical lines indicate the $90 \%$ confidence interval of estimated completeness while points indicate the midpoint of that interval. c, Completeness as estimated by viralComplete, based on the ratio of the contig length to the length of the classified reference genome. d, VIBRANT quality tiers. e-g, Benchmarking CheckV completeness for genome fragments derived from NCBI GenBank genomes. Estimation error is shown for viruses according to their Baltimore classification (e), cellular host (f) and viral family ( $\mathbf{g}$ ). Only medium- and high-confidence AAl-based estimates are shown. Viral categories representing at least 30 viruses are indicated on the vertical axes. For box plots, the middle line denotes the median, the box denotes the IQR and the whiskers denote 1.5×IQR. RT, reverse transcribing; ssDNA, single-stranded DNA.

First, we used CheckV to identify three types of complete genome from IMG/VR, including DTR contigs $(n=15,211)$, proviruses with 5 ' and 3 ' host regions $(n=451)$ and contigs with ITRs $(n=624)$. The longest DTR contig we identified was a 528,258-bp sequence from a saline lake in Antarctica estimated to be $100.0 \%$ complete and supported by paired-end reads that connected contig ends. Based on gene content and phylogeny, this sequence is probably a novel member of one of the recently defined clades of 'huge' phages ${ }^{42}$ (Supplementary Text and Extended Data Fig. 5). To validate the other potentially complete genomes, we compared the contigs to CheckV's database of complete reference genomes based on AAI, estimated completeness (excluding low-confidence estimates) and identified high-quality assemblies (that is, $>90 \%$ complete). We found that $90.0 \%$ of the DTR contigs with estimated completeness met the high-quality standard compared to only $46.4 \%$ of complete proviruses and $33.2 \%$ of ITRs (Extended Data Fig. 6). In the case of proviruses, lower estimated completeness may be due to their domestication and degradation in the host genome over time ${ }^{43}$ or, in rare cases, due to false positives. Nonetheless, predictions from all three methods were highly enriched in high-quality genomes compared with other contigs from IMG/VR. These results further confirm that DTRs are a good indicator of complete viral genomes most of the time $\mathrm{e}^{33}$, but suggest that greater caution is needed when interpreting other signatures.

Next, we used CheckV to estimate completeness for the entire IMG/VR dataset, including genome fragments. Using the accurate AAI-based approach, completeness could be estimated for $80.2 \%$ of IMG/VR contigs with high or medium confidence, including $84.5 \%$ from host-associated, $83.9 \%$ from marine, $75.1 \%$ from freshwater and $70.0 \%$ from soil environments. For the majority of these contigs, the best hit in the CheckV database was a DTR sequence $(n=501,055,85.0 \%)$ and was often derived from the same habitat as the IMG/VR contig (Extended Data Fig. 7). We next applied the HMM-based approach to estimate the completeness range for novel IMG/VR contigs lacking confident AAI-based estimates, increasing the percentage of contigs with estimated completeness to 97.9. AAIand HMM-based estimates were well correlated for contigs having both predictions (Spearman's $\rho=0.90$ ), with AAI-based predictions often falling within the completeness range predicted by the HMM approach (Extended Data Fig. 8).

We next classified IMG/VR sequences into quality tiers according to their estimated completeness, revealing $1.9 \%$ complete, $2.4 \%$ high-, $6.4 \%$ medium- and $87.3 \%$ low-quality sequences, with the remaining $2.0 \%$ of undetermined quality (Fig. $5 \mathrm{a}$ ). Contig sizes were strongly correlated with quality tiers, with complete genomes centered at $44 \mathrm{~kb}$, which is consistent with genome sizes from the order Caudovirales (Fig. 5b). In a small number of cases, IMG/VR contigs were considerably longer than expected (for example, 290 contigs more than twofold expected length based on AAI) and which, upon inspection of $k$-mer frequencies, revealed the same genome repeated multiple times (2.0× to $6.8 \times)$ (Extended Data Fig. 9). While these are probably artifacts of metagenomic assembly, they are easily identified and less common than previously suggested ${ }^{42}$. 

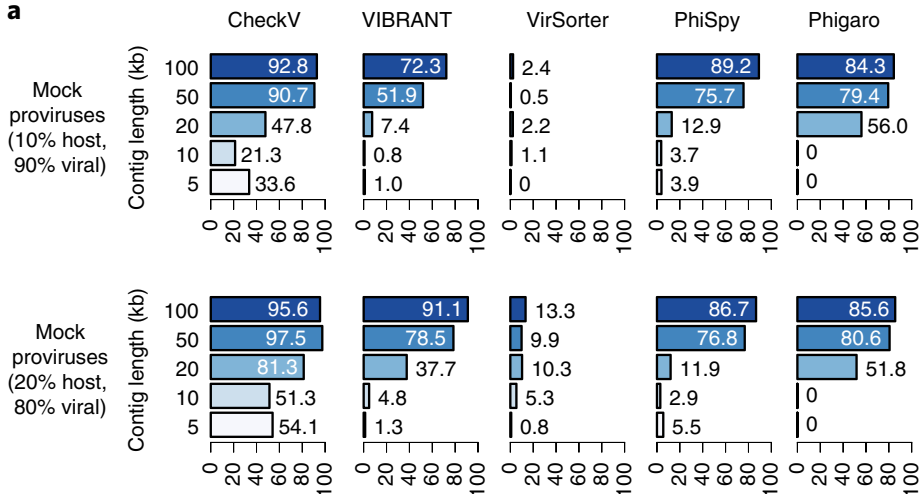

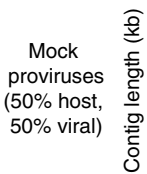
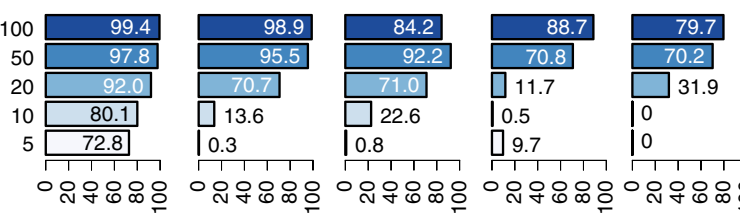

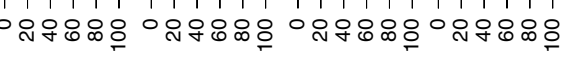

Percentage classified as provirus (viral region covering $<95 \%$ of fragment length)

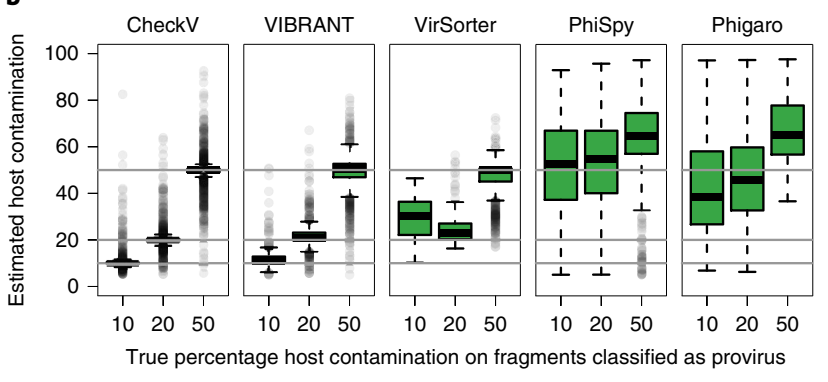

C

Mock viral fragments (5-100 kb, 100\% viral)

d IMG/VR circular viral contigs
(>20-bp DTR, $>90 \%$ completenes

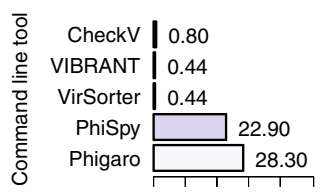

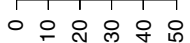

Percentage classified as provirus

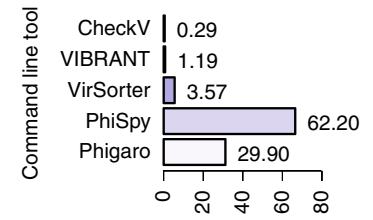

Percentage classified as provirus

Fig. 4 | Sensitivity and specificity of predicting host regions on viral contigs. a-c, Mock proviruses were generated for 382 paired bacteriophage-host pairs. Random genome fragments were extracted from mock proviruses at various lengths and levels of host contamination and used as input to CheckV and other tools. A fragment was classified by a tool as a provirus if it contained a predicted viral region that covered $<95 \%$ of the fragment length.

a, Sensitivity of Check $V$ and other tools in detecting host contamination on proviral fragments with at least $10 \%$ host contamination $(n=4,593)$.

b. Estimated contamination versus true contamination for correctly classified provirus fragments. For box plots, the middle line denotes the median, the box denotes the IQR and the whiskers denote 1.5 $\times$ IQR. c, To determine specificity, CheckV and other tools were used to predict host regions on entirely viral fragments $(n=1,367)$. d, CheckV and other tools were used to predict host regions on circular viral contigs from IMG/VR $(n=1,345)$.

We also applied CheckV to the Global Ocean Virome (GOV) 2.0 dataset $^{6}$ (Supplementary Table 12), which revealed remarkably similar patterns (Extended Data Fig. 10). Like IMG/VR, the GOV dataset contains viral contigs that are at least $5 \mathrm{~kb}$ but, unlike IMG/VR, the original samples were derived from the open ocean and enriched for viral particles before sequencing. We identified a combined total of 44,652 complete or high-quality genomes across both datasets, but these represented a mere $3.6 \%$ of the total number of contigs. The highly fragmented nature of sequences from IMG/ VR and GOV probably reflects numerous challenges in the assembly of viruses from short-read metagenomes, including repetitive regions $^{12}$, strain heterogeneity ${ }^{13}$, low-abundance viral populations ${ }^{33}$ and low sample biomass ${ }^{44}$. Long-read sequencing circumvents many of these challenges and has recently been used to obtain high-quality viral genomes without the need for metagenomic assembly ${ }^{12,13}$.

Using CheckV to discriminate viral-encoded functions from host contamination. Finally, we used CheckV to identify putative proviruses from the IMG/VR database that were flanked on one or both sides by host genes. Overall, only 17,057 contigs followed this pattern (Fig. 5c) with $96.5 \%$ of host regions occurring on only one side and typically representing a minor fraction of contig length (average, 26.8\%; Fig. 5d). Proviruses were detected in all biomes, although more frequently in host-associated metagenomes. Longer contigs were considerably more likely to contain a host region (Fig. 5d), which is probably explained by the higher sensitivity of CheckV for longer sequences and a greater chance of intersecting a host-provirus boundary. Supporting these predictions, the majority of long proviruses ( $>50 \mathrm{~kb}$ with $>20 \%$ contamination, $n=783$ ) were confirmed by either VirSorter or VIBRANT (76.8\%) and contained integrases $(85.2 \%)$. We also used CheckV to identify proviruses in the GOV dataset, revealing similar patterns (Extended Data Fig. 10). Together, these results confirm that the majority of
IMG/VR and GOV sequences are entirely viral or encode a short, host-derived region.

Notably, even a small amount of contamination by host-derived sequences can impair downstream analyses, especially those related to the gene content and functional potential of uncultivated viruses $^{18}$. To illustrate this potential issue, we functionally annotated the 17,057 IMG/VR proviruses using the Kyoto Encyclopedia of Genes and Genomes (KEGG) database ${ }^{45}$ and compared the functions of genes in host versus viral regions. Overall, host regions represented only $19.2 \%$ of the genes but $59.7 \%$ of genes assigned to a KEGG metabolic pathway (Fig. 5f). Several pathways were highly enriched in host genes, including those for biosynthesis of antibiotics, carbon metabolism and ABC transporters (Fig. $5 \mathrm{~g}$ and Supplementary Table 12). For example, 254 provirus genes were annotated as multidrug efflux pumps or multidrug resistance proteins, but $95.3 \%$ of these were found in host regions. In contrast, KEGG pathways for recombination, mismatch repair and nucleotide biosynthesis were all highly enriched in viral regions. Without the detection of provirus boundaries provided by CheckV, it would not have been possible to discriminate true viral-encoded functions from host contamination except through manual curation, which becomes nearly impossible for large datasets like IMG/VR.

\section{Discussion}

Here we have presented CheckV, an automated pipeline used for assessing the quality of single-contig viral genomes, along with an expanded database of complete viral genomes that we systematically identified from environmental data sources. We anticipate that CheckV will be broadly useful in future viral metagenomics studies and for reporting quality statistics required in the MIUViG checklist ${ }^{17}$. Estimation of completeness will be especially valuable in distinguishing near-complete genomes from short genome fragments, as these two types of sequence are associated with dif- 
a

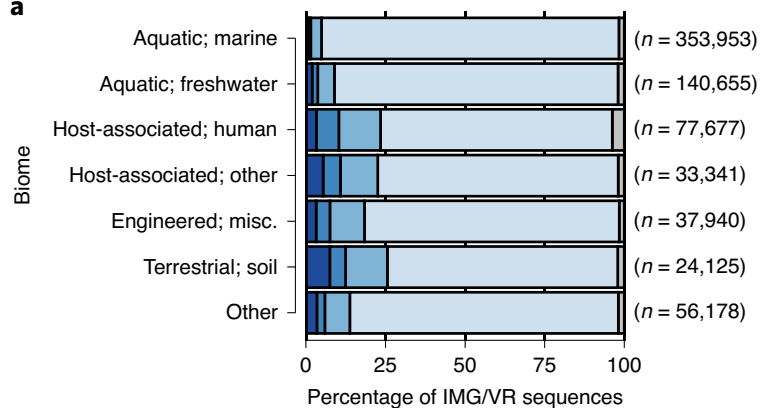

b

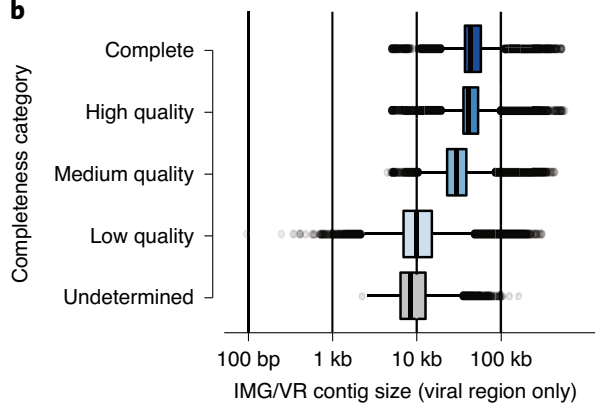

Estimated completeness

Complete

\begin{tabular}{l|l} 
MIUViG: \\
high quality
\end{tabular}

50-90\% complete

MIUViG

\begin{tabular}{l|l} 
genome \\
fragment
\end{tabular}

Undetermined
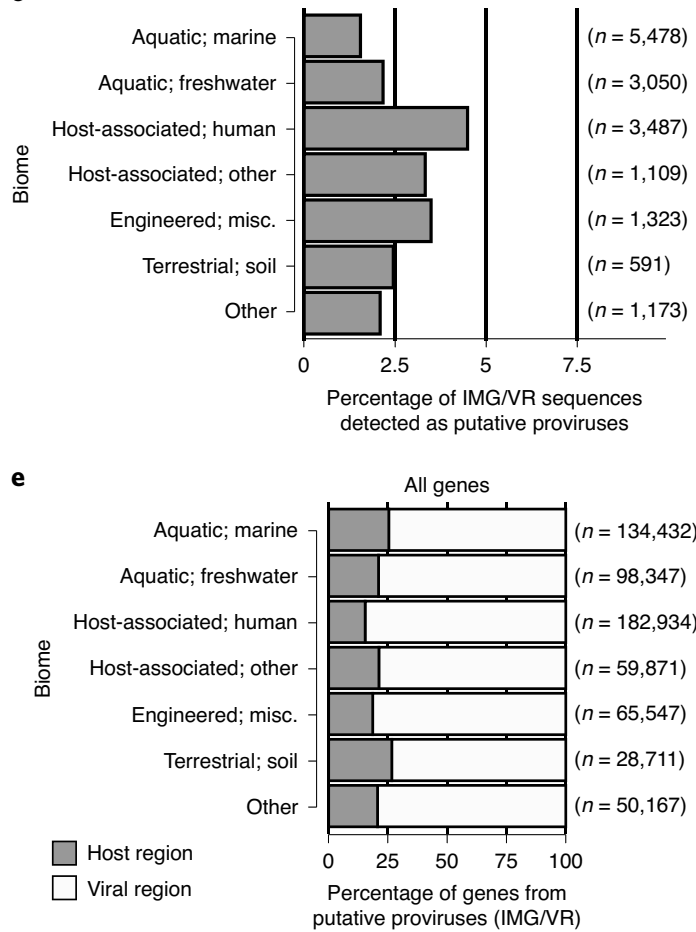
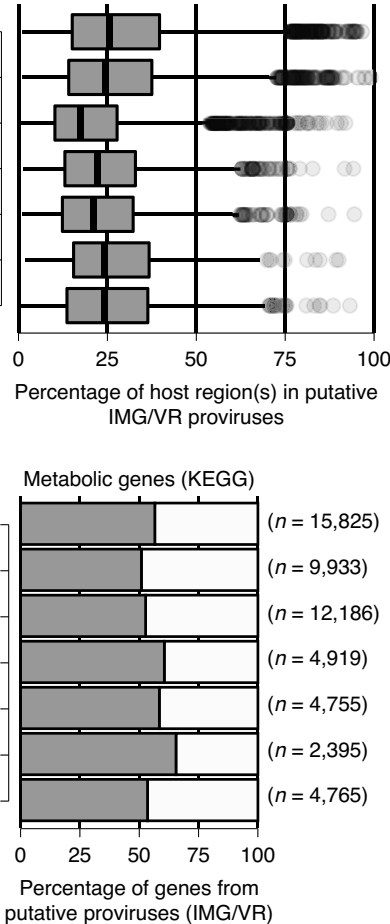

d

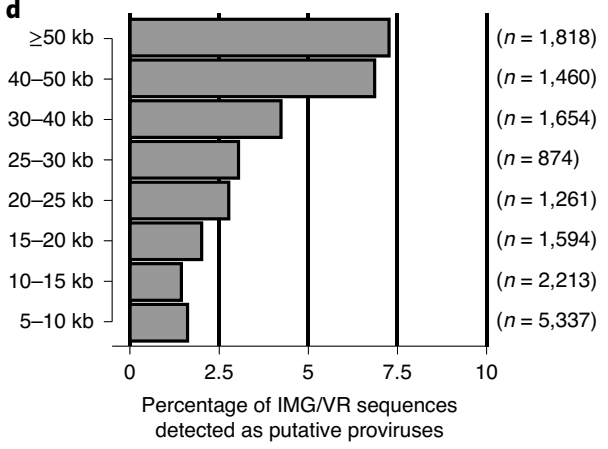

f

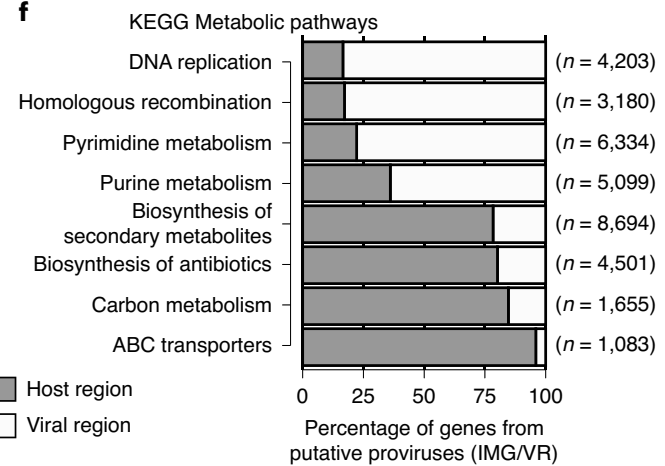

Fig. 5 | Application of CheckV to the IMG/VR database. a, Estimated completeness of IMG/VR contigs by biome. b, Distribution of IMG/VR contig length across quality tiers: complete $(n=13,700)$, high quality $(n=16,544)$, medium quality $(n=45,109)$, low quality $(n=634,117)$ and undetermined $(n=14,399)$.

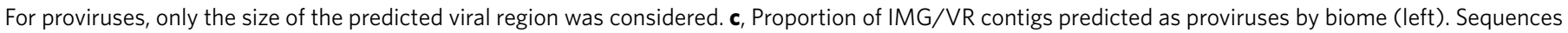

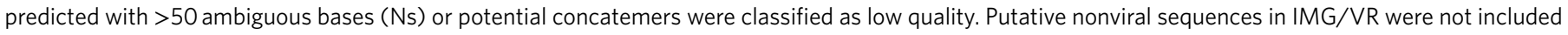
( $>5$ host genes and $>2 \times$ host versus viral genes). Length of the predicted host region by biome for IMG/VR contigs predicted as proviruses (right). Region length is indicated as a percentage of total contig length. $\mathbf{d}$, Proportion of contigs predicted as proviruses by contig length. $\mathbf{e}$, Percentage of all genes from predicted proviruses found in viral/host regions (left). Percentage of metabolic genes from predicted proviruses found in viral/host regions (right).

f, Percentage of genes from selected KEGG pathways for predicted proviruses found in viral/host regions. For box plots, the middle line denotes the median, the box denotes the IQR and the whiskers denote 1.5× IQR. Misc., miscellaneous.

ferent limitations and biases. For example, the inclusion of small genome fragments may result in inflated estimates of viral diversity based on genome clustering due to insufficient overlap between sequences. Meanwhile, the removal of genes originating from the host genome will be critically important in reducing false positives in viral studies focusing on auxiliary metabolic genes or the discovery of novel protein families. We also expect that CheckV's database of complete viral genomes will be a useful community resource that contains a wealth of untapped insights into novel viruses from diverse environments.

Several improvements in CheckV may be possible in the future. First, it will be important to incorporate new viral genomes as these become available, to continually expand the environmental and taxonomic diversity of the reference database. Inclusion of novel RNA viruses and eukaryotic viruses will be especially valuable, as these types of genome are currently under-represented in the database.
Second, metagenomic read mapping could be used for a variety of inferences, including identification of circular contigs ${ }^{21}$, refinement of virus-host boundaries and determination of genome termini ${ }^{46}$. Third, viral MAGs (that is, derived from metagenome binning) and segmented viral genomes, which are represented by multiple sequences, pose several additional challenges not addressed here, including the presence of contamination from other viruses or cellular organisms. Finally, CheckV could be adapted to detect other artifacts, such as chimeras resulting from the assembly of closely related viruses or nonviral sequences resulting from false-positive viral predictions.

\section{Online content}

Any methods, additional references, Nature Research reporting summaries, source data, extended data, supplementary information, acknowledgements, peer review information; details of 
author contributions and competing interests; and statements of data and code availability are available at https://doi.org/10.1038/ s41587-020-00774-7.

Received: 19 May 2020; Accepted: 12 November 2020; Published online: 21 December 2020

\section{References}

1. Shkoporov, A. N. \& Hill, C. Bacteriophages of the human gut: the "Known Unknown" of the microbiome. Cell Host Microbe 25, 195-209 (2019).

2. Williamson, K. E. et al. Viruses in soil ecosystems: an unknown quantity within an unexplored territory. Annu. Rev. Virol. 4, 201-219 (2017).

3. Breitbart, M. et al. Phage puppet masters of the marine microbial realm. Nat. Microbiol. 3, 754-766 (2018).

4. Koonin, E. V. et al. Global organization and proposed megataxonomy of the virus world. Microbiol. Mol. Biol. Rev. 84, e00061-19

5. Paez-Espino, D. et al. Uncovering Earth's virome. Nature 536, 425-430 (2016).

6. Gregory, A. C. et al. Marine DNA viral macro- and microdiversity from pole to pole. Cell 177, 1109-1123 (2019).

7. Gregory, A. C. et al. The gut virome database reveals age-dependent patterns of virome diversity in the human gut. Cell Host Microbe 28, 724-740 (2020).

8. Emerson, J. B. et al. Host-linked soil viral ecology along a permafrost thaw gradient. Nat. Microbiol. 3, 870-880 (2018).

9. Ren, J. et al. VirFinder: a novel $k$-mer based tool for identifying viral sequences from assembled metagenomic data. Microbiome 5, 69 (2017).

10. Roux, S. et al. VirSorter: mining viral signal from microbial genomic data. PeerJ 3, e985 (2015).

11. Kieft, K., Zhou, Z. \& Anantharaman, K. VIBRANT: automated recovery, annotation and curation of microbial viruses, and evaluation of viral community function from genomic sequences. Microbiome 8, 90 (2020).

12. Beaulaurier, J. et al. Assembly-free single-molecule sequencing recovers complete virus genomes from natural microbial communities. Genome Res. 30, 437-446 (2020).

13. Warwick-Dugdale, J. et al. Long-read viral metagenomics captures abundant and microdiverse viral populations and their niche-defining genomic islands. PeerJ 7, e6800 (2019).

14. Suzuki, Y. et al. Long-read metagenomic exploration of extrachromosomal mobile genetic elements in the human gut. Microbiome 7, 119 (2019).

15. Schulz, F. et al. Giant virus diversity and host interactions through global metagenomics. Nature 578, 432-436 (2020).

16. Smits, S. L. et al. Assembly of viral genomes from metagenomes. Front. Microbiol. 5, 714 (2014).

17. Roux, S. et al. Minimum Information about an uncultivated virus genome (MIUViG). Nat. Biotechnol. 37, 29-37 (2019).

18. Roux, S. et al. Assessment of viral community functional potential from viral metagenomes may be hampered by contamination with cellular sequences. Open Biol. 3, 130160 (2013).

19. Belyi, V. A., Levine, A. J. \& Skalka, A. M. Sequences from ancestral single-stranded DNA viruses in vertebrate genomes: the Parvoviridae and Circoviridae are more than 40 to 50 million years old. J. Virol. 84, 12458-12462 (2010)

20. Philippe, N. et al. Pandoraviruses: amoeba viruses with genomes up to 2.5 $\mathrm{Mb}$ reaching that of parasitic eukaryotes. Science 341, 281-286 (2013).

21. Chung, C. H. et al. Predicting genome terminus sequences of Bacillus cereus-group bacteriophage using next generation sequencing data. BMC Genomics 18, 350 (2017).

22. Antipov, D. et al. Metaviral SPAdes: assembly of viruses from metagenomic data. Bioinformatics 36, 4126-4129 (2020).

23. Akhter, S., Aziz, R. K. \& Edwards, R. A. PhiSpy: a novel algorithm for finding prophages in bacterial genomes that combines similarity- and composition-based strategies. Nucleic Acids Res. 40, e126 (2012).

24. Starikova, E. V. et al. Phigaro: high-throughput prophage sequence annotation. Bioinformatics 36, 3882-3884 (2020).

25. Paez-Espino, D. et al. IMG/VR v.2.0: an integrated data management and analysis system for cultivated and environmental viral genomes. Nucleic Acids Res. 47, D678-D686 (2019).

26. Coutinho, F. H., Edwards, R. A. \& Rodriguez-Valera, F. Charting the diversity of uncultured viruses of archaea and bacteria. BMC Biol. 17, 109 (2019).
27. Hindmarsh, P. \& Leis, J. Retroviral DNA integration. Microbiol. Mol. Biol. Rev. 63, 836-843 (1999).

28. Tisza, M. J. et al. Discovery of several thousand highly diverse circular DNA viruses. eLife https://doi.org/10.7554/eLife.51971 (2020).

29. Casjens, S. R. \& Gilcrease, E. B. Determining DNA packaging strategy by analysis of the termini of the chromosomes in tailed-bacteriophage virions. Methods Mol. Biol. 502, 91-111 (2009).

30. Munoz-Lopez, M. \& Garcia-Perez, J. L. DNA transposons: nature and applications in genomics. Curr. Genomics 11, 115-128 (2010).

31. Yan, Z. et al. Inverted terminal repeat sequences are important for intermolecular recombination and circularization of adeno-associated virus genomes. J. Virol. 79, 364-379 (2005).

32. Savilahti, H. \& Bamford, D. H. Linear DNA replication: inverted terminal repeats of five closely related Escherichia coli bacteriophages. Gene 49, 199-205 (1986).

33. Roux, S. et al. Benchmarking viromics: an in silico evaluation of metagenome-enabled estimates of viral community composition and diversity. PeerJ 5, e3817 (2017).

34. Sayers, E. W. et al. GenBank. Nucleic Acids Res. 48, D84-D86 (2020).

35. Chen, I. A. et al. IMG/M v.5.0: an integrated data management and comparative analysis system for microbial genomes and microbiomes. Nucleic Acids Res. 47, D666-D677 (2019).

36. Mitchell, A. L. et al. MGnify: the microbiome analysis resource in 2020. Nucleic Acids Res. 48, D570-D578 (2020).

37. Nayfach, S. et al. New insights from uncultivated genomes of the global human gut microbiome. Nature 568, 505-510 (2019).

38. Pasolli, E. et al. Extensive unexplored human microbiome diversity revealed by over 150,000 genomes from metagenomes spanning age, geography, and lifestyle. Cell 176, 649-662 (2019).

39. Soto-Perez, P. et al. CRISPR-Cas system of a prevalent human gut bacterium reveals hyper-targeting against phages in a human virome catalog. Cell Host Microbe 26, 325-335 (2019).

40. Yutin, N. et al. Eukaryotic large nucleo-cytoplasmic DNA viruses: clusters of orthologous genes and reconstruction of viral genome evolution. Virol. J. 6, 223 (2009).

41. Parks, D. H. et al. A standardized bacterial taxonomy based on genome phylogeny substantially revises the tree of life. Nat. Biotechnol. 36, 996-1004 (2018).

42. Al-Shayeb, B. et al. Clades of huge phages from across Earth's ecosystems. Nature 578, 425-431 (2020).

43. Bobay, L. M., Touchon, M. \& Rocha, E. P. Pervasive domestication of defective prophages by bacteria. Proc. Natl Acad. Sci. USA 111, 12127-12132 (2014).

44. Rinke, C. et al. Validation of picogram- and femtogram-input DNA libraries for microscale metagenomics. PeerJ 4, e2486 (2016).

45. Kanehisa, M. \& Goto, S. KEGG: Kyoto Encyclopedia of Genes and Genomes. Nucleic Acids Res. 28, 27-30 (2000).

46. Garneau, J. R. et al. PhageTerm: a tool for fast and accurate determination of phage termini and packaging mechanism using next-generation sequencing data. Sci. Rep. 7, 8292 (2017).

47. Mukherjee, S. et al. Genomes OnLine database (GOLD) v.7: updates and new features. Nucleic Acids Res. 47, D649-D659 (2019).

48. Mauri, M. et al. RAWGraphs: A visualisation platform to create open outputs. in Proc. 12th Biannual Conference on Italian SIGCHI 1-5 (2017).

Publisher's note Springer Nature remains neutral with regard to jurisdictional claims in published maps and institutional affiliations.

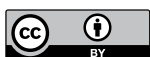

Open Access This article is licensed under a Creative Commons

Attribution 4.0 International License, which permits use, sharing, adaptation, distribution and reproduction in any medium or format, as long as you give appropriate credit to the original author(s) and the source, provide a link to the Creative Commons license, and indicate if changes were made. The images or other third party material in this article are included in the article's Creative Commons license, unless indicated otherwise in a credit line to the material. If material is not included in the article's Creative Commons license and your intended use is not permitted by statutory regulation or exceeds the permitted use, you will need to obtain permission directly from the copyright holder. To view a copy of this license, visit http://creativecommons. org/licenses/by/4.0/.

(C) The Author(s) 2020 


\section{Methods}

Database of HMMs for classification of viral and microbial genes. We selected HMMs from existing databases that could be leveraged to classify genes as either viral or microbial with high specificity. First, 125,754 HMMs were downloaded from seven databases: VOGDB (release 97, $n=25,399$, http://vogdb.org), IMG/VR (downloaded January 2020, $n=25,281)^{25}$, RVDB (release 17, $\left.n=9,911\right)^{49}$, KEGG Orthology (release 2October 2019, $n=22,746)^{45}$, Pfam A (release 32, $\left.n=17,929\right)^{50}$, Pfam B (release 27, $n=20,000)^{51}$ and TIGRFAM (release 15, $\left.n=4,488\right)^{52}$. Next, we used hmmsearch v.3.1b2 (ref. ${ }^{53}$ ) to align the HMMs versus 1,590,764 proteins from 30,903 NCBI GenBank viral genomes (downloaded 1 June 2019) ) $^{34}$ and 5,749,148 proteins from 2,015 bacterial and 239 archaeal genomes from GTDB, release 89). For computational reasons, we selected a maximum of one genome per GTDB family and, when multiple genomes were available, we chose the one with the highest CheckM quality score (completeness $-5 \times$ contamination). Additionally, we ran VIBRANT v.1.2.0 (ref. ${ }^{11}$ ), VirSorter v.1.0.5 (ref. ${ }^{10}$ ) and PhiSpy v.3.7.8 (ref. ${ }^{23}$ ) using default parameters to identify and remove 590,484 viral proteins identified on proviruses in the selected GTDB genomes.

Based on the hmmsearch results, we calculated the percentage of viral and microbial genes matching each HMM at bit-score cutoffs ranging from 25 to 1,000 , in increments of 5 . We then selected the lowest bit-score cutoff for each HMM that resulted in a difference $>100$-fold between the percentage of the total viral gene set and that of the total microbial gene set matched by the HMM (that is, bit-score cutoff for which the hits were strongly enriched in either virus or microbial genes). To limit false positives, we excluded HMMs that were classified as microbial specific but were derived from primarily viral databases (VOGDB, IMG/VR, RVDB) or contained viral terms (viral, virus, virion, provirus, capsid, terminase) for HMMs from other databases. Using this approach, 114,765 HMMs were identified as either viral specific or microbial specific.

Next, we selected the maximally informative subset of HMMs to reduce the size of the database and limit CheckV computing time. First, we retained 44,415 HMMs with at least 20 viral hits or at least 100 microbial hits after applying the bit-score cutoffs. Next, we calculated the Jaccard similarity between all pairs of HMMs based on each HMMs set of gene hits. For computational efficiency, we used the 'all_pairs' function in the SetSimilaritySearch Python package (https:// github.com/ekzhu/SetSimilaritySearch). Jaccard similarities were used as input for single-linkage clustering with a Jaccard similarity cutoff of 0.5 , resulting in 15,958 nonredundant HMMs (8,773 viral specific, 7,185 microbial specific). To form the final database, we selected the HMM with the greatest number of gene hits from each cluster of HMMs.

Identification of virus-host boundaries. Given a viral contig, CheckV predicts host-virus boundaries in three stages.

First, proteins are predicted using Prodigal v.2.6.3 (option '-p meta' for metagenome mode) $)^{54}$ and compared to the 15,958 HMMs using hmmsearch. Each protein is classified as viral, microbial or unannotated according to its top-scoring hit after applying the HMM-specific bit-score cutoffs. Viral- and microbial-annotated genes are assigned a viral score of +1 and -1 , respectively. Additionally, the GC content of each gene is calculated (range, 0-100).

Second, CheckV scans across the contig and quantifies differences in the viral score (that is, +1 or -1 ) and GC content between a pair of adjacent gene windows. The 5 ' gene window extends to the left contig endpoint, and the 3 ' gene window is sized to contain $30 \%$ of genes on the contig with no fewer than 15 genes and no more than 50 genes. The $3^{\prime}$ window may contain fewer then 15 genes if it ends at the right contig endpoint. CheckV then computes a breakpoint score, $S$, based on the absolute difference in the average viral score, $V$, and average GC content, $G$, between genes in the $5^{\prime}$ and $3^{\prime}$ windows: $S=\left|V_{5^{\prime}}-V_{3^{\prime}}\right|+0.02 \times\left|G_{5^{\prime}}-G_{3^{3}}\right|$. Unannotated genes are not included when calculating $V$. The value of $S$ ranges from 0 to 4 , given that $\left|V_{5^{\prime}}-V_{3^{\prime}}\right|$ and $0.02 \times\left|G_{5^{\prime}}-G_{3^{\prime}}\right|$ both range from 0 to 2 . CheckV also stores the orientation of each breakpoint (that is, host-virus or virus-host) based on the values of $V_{5^{\prime}}$ and $V_{3^{\prime}}$. These scores are computed at each intergenic position, moving from the 5 ' end to the 3 ' end of the contig.

Third, CheckV identifies breakpoints based on the following rules: $S \geq 1.2$, $\geq 30 \%$ genes annotated as microbial in the host region, $\geq 2$ microbial-annotated genes in the host region and $\geq 2$ viral-annotated genes in the viral region. For very short contigs (fewer than ten genes), CheckV requires only one microbial-annotated gene in the host region and one viral-annotated gene in the viral region. After these filters, CheckV chooses the first encountered breakpoint with the highest score. After selecting the first breakpoint, CheckV then repeats the steps listed above to search for additional breakpoints, using the last identified breakpoint as the new starting position for the 5 ' gene window. The algorithm ends when no new breakpoints are found. Algorithm parameters were fine-tuned empirically based on a dataset of mock proviruses and sequences from the IMG/VR database.

AAI-based estimation of genome completeness. Given a viral contig, CheckV estimates genome completeness in four stages. First, it performs an amino acid alignment of Prodigal-predicted protein-coding genes from the contig against the database of reference genomes using DIAMOND v.0.9.30 (ref. ${ }^{55}$ ), with the option '-evalue 1e-5-query-cover 50 --subject-cover 50 -k 10000'. Based on these alignments, the following metrics are computed for the viral contig versus each reference genome: AAI: length-weighted average identity across aligned proteins; alignment fraction (AF): percentage of amino acids aligned from the query sequence; and alignment score: AAI $\times$ AF. Second, CheckV identifies the top hit in the database for the contig (that is, the reference genome with the highest alignment score) and all reference genomes with alignment scores within $50 \%$ of the top hit. The expected genome length of the viral contig, $\hat{G}$, is then estimated by taking a weighted average of the genome sizes of matched reference genomes, where the alignment scores are used as weights. Reference genome lengths are further weighted based on their source: 2.0 for isolate viruses and 1.0 for metagenome-derived viruses, which are more likely to contain assembly errors and artifacts. CheckV also reports the confidence level of this estimate (low, medium or high), which is determined based on the length of the viral contig and the alignment score to the top reference genome (see Confidence levels for AAI-based completeness estimates for the method used to estimate confidence levels). Third, CheckV estimates the genome completeness of each viral contig, $\hat{C}$, using the formula: $\hat{C}=100 \times L / \hat{G}$, where $L$ is the length of the viral region for proviruses, or the contig length otherwise.

HMM-based estimation of genome completeness. An HMM-based approach was developed to estimate completeness for novel viruses that are too diverged from CheckV genomes to obtain an accurate AAI-based estimate. First, CheckV identifies viral genes on the contig based on comparison to the 8,773 viral HMMs (see 'Identification of virus-host boundaries' above). Each viral HMM is associated with one or more reference genomes and this information is stored in the database, as well as the coefficient of variation, which is a measure of the variability in reference genome length associated with each HMM. For each HMM on a viral contig, CheckV identifies the range of completeness values corresponding to the fifth and 95th percentiles of the distribution of reference genome length containing the same HMM (for example, 35-65\% completeness). In theory, we expect the true completeness to be greater than the lower bound $95 \%$ of the time, below the upper bound $95 \%$ of the time and between both bounds $90 \%$ of the time. In practice, however, these outcomes are less frequent due to error in the underlying estimates. CheckV performs this step for each HMM, resulting in a distribution of completeness ranges for each contig (for example, 45-67, 35-55 and 42-49\%). Finally, CheckV takes a weighted average of the ranges, where the weights are equal to the inverse of the coefficient of variation with a maximum value of 50 . Therefore, HMMs with a low coefficient of variation (which are associated with genomes of consistent length) receive higher weight.

Confidence levels for AAI-based completeness estimates. We conducted a large-scale benchmarking experiment to derive confidence levels for AAI-based completeness estimation. First, we extracted a random fragment from each of CheckV's reference genomes to simulate metagenomic contigs of varying length (200 and $500 \mathrm{bp}$ and 1, 2, 5, 10, 20 and $50 \mathrm{~kb}$ ). Next, we used CheckV to compute the alignment score between each contig and each complete genome in the reference database. We then compared the true genome length of each contig (that is, the length before fragmentation), $L$, to the estimated genome length based on each matched reference genome, $\hat{L}$, and computed the relative unsigned error, as $100 \times|L-\hat{L}| / L$. We then computed the median relative unsigned error after grouping the estimates based on their alignment score and contig length. Finally, we determined three confidence levels: high confidence ( $0-5 \%$ median unsigned error), medium confidence (5-10\% median unsigned error) and low confidence ( $>10 \%$ median unsigned error). Using this information, CheckV reports a confidence level in the estimated completeness value for each input contig based on contig length and alignment score (that is, a combination of AAI and AF) to the top database hit. By default, only medium- and high-confidence estimates are included in the final report.

Database of complete viral genomes for AAI-based completeness estimation. We downloaded 30,903 genomes from NCBI GenBank on 1 June 2019, excluding 1,937 that were indicated as 'partial,' 'chimeric' or 'contaminated'. Of the remaining $28,966,677$ (2.3\%) were labeled as 'metagenomic' or 'environmental', suggesting that the vast majority are derived from cultivated isolates.

Next, we used CheckV to systematically search for complete genomes of uncultivated viruses from publicly available and previously assembled metagenomes, metatranscriptomes and metaviromes. An assembled contig was considered complete if it was at least 2,000 bp in length and included a DTR of at least $20 \mathrm{bp}$ (DTR contigs). We searched for DTR contigs in the following datasets: 19,483 metagenomes and metatranscriptomes from IMG/M (accessed September 2019) 35 $^{35}$ 11,752 metagenomes from MGnify (accessed 16 April 2019) ${ }^{36}$, 9,428 metagenomes assembled by Pasolli et al..$^{38}$, an expanded collection of 4,763 metagenomes from the HGM dataset ${ }^{37}, 1,831$ viromes from $\mathrm{HuVirDB}^{39}$ and 145 viromes from the Global Ocean Virome 2.0 dataset $^{6}$.

From this initial search, we identified a total of 751,567 DTR contigs. To minimize false positives and other artifacts, we removed the following: (1) 45,448 contigs with low-complexity repeats (for example, AAAAA...), as determined by dustmasker from the BLAST+ package v.2.9.0 (ref. $\left.{ }^{56}\right) ;$; (2) 11,359 contigs classified as proviral by CheckV; (3) 5,737 contigs with repeats 
occurring more than five times per contig, which could represent repetitive genetic elements such as clustered regularly interspaced short palindromic repeat (CRISPR) arrays; (4) 6,543 contigs that contained a large duplicated region spanning $\geq 20 \%$ of the contig length, resulting from rare instances where assemblers concatenate multiple copies of the same genome; and (5) 1,293 contigs containing $\geq 1 \%$ ambiguous base calls. After application of these filters, 686,030 contigs remained ( $91.3 \%$ of the total).

Next, we used a combination of CheckV marker genes and VirFinder ${ }^{9}$ to classify 116,666 DTR contigs as viral. First, the DTR contigs were used as input to VirFinder v.1.1 with default parameters, and to CheckV to identify viral and microbial marker genes. We additionally searched for genes related to plasmids and other nonviral mobile genetic elements using a database of $141 \mathrm{HMMs}$ from recent publications $\mathrm{s}^{57-59}$. A contig was classified as viral if the number of viral genes exceeded that of microbial and plasmid genes $(n=99,345)$, or VirFinder reported a $P<0.01$ with no plasmid genes and no more than one identified microbial gene $(n=36,084)$.

\section{Taxonomic annotation of CheckV reference genomes. Annotations} were determined based on HMM searches against a custom database of 1,000 taxonomically informative HMMs from the VOG database (http://vogdb. org/). These HMMs were selected for major bacterial and archaeal viral groups with consistent genome length and at least ten representative genomes, including: Caudovirales, CRESS-DNA and Parvoviridae, Autolykiviridae, Fusello- and Guttaviridae, Inoviridae, Ligamenvirales Ampulla-Bicauda- and Turriviridae, Microviridae and Riboviria. For each group, VOGs found in $\geq 10 \%$ of the group members and never detected outside of this group were considered as marker genes. All CheckV reference genomes were annotated based on the clade with the most HMM hits. Overall, 96.4\% of HMM hits were to a single viral taxon.

Validating the completeness of CheckV reference genomes. Next, we validated the completeness for all GenBank genomes and DTR contigs. First, we used CheckV to estimate the completeness for all sequences after excludsion of self-matches. This was performed using a database of GenBank sequences only and another of DTR contigs only. Any sequence with $<90 \%$ estimated completeness using either database was excluded (medium- and high-confidence estimates only). Second, we compared genome length to the known distribution of genome length for the annotated viral taxon (for example, Microviridae). Any genome considered an outlier or shorter than the shortest reference genome for the annotated clade was excluded. After application of these exclusion filters, we then selected genomes for inclusion with $\geq 90 \%$ estimated completeness using either database (mediumand high-confidence estimates only) or $>30 \mathrm{~kb}$ without a completeness estimate. These selection criteria were chosen to minimize the number of false positives (that is, genome fragments wrongly considered complete genomes) at the cost of some false negatives (that is, removal of truly complete genomes). This resulted in 24,834 GenBank genomes and 76,262 DTR contigs that were used to form the final CheckV genome database.

Generating a nonredundant set of CheckV reference genomes. Average nucleotide identity (ANI) and alignment fraction (AF) were computed between the 24,834 GenBank genomes and 76,262 DTR contigs using a custom script. Specifically, we used blastn from the BLAST+ package v.2.9.0 (option: perc identity $=90$ max_target_seqs $=10000)$ to generate local alignments between all pairs of genomes. Based on this, we estimated ANI as the average DNA identity across alignments after weighting the alignments by length. The AF was computed by taking the total length of merged alignment coordinates and dividing this by the length of each genome. Clustering was then performed using a greedy, centroid-based algorithm in which (1) genomes were sorted by length, (2) the longest genome was designated as the centroid of a new cluster, (3) all genomes within 95\% ANI and 85\% AF were assigned to that cluster and (4) steps 2 and 3 were repeated until all genomes had been assigned to a cluster, resulting in 52,141 nonredundant genomes.

Benchmarking estimation of genome completeness. To benchmark genome completeness estimates, we used 2,000 uncultivated, complete viral genomes from IMG/VR (>20-bp DTR). We used IMG/VR genomes, because these are derived from diverse habitats and represent highly novel sequences. After removal of terminal repeats, a single genome fragment was randomly extracted from each IMG/VR genome (1-100\% completeness). These sequences were used as input to CheckV, VIBRANT v.1.2.0 (ref. ${ }^{11}$ ) and viralComplete ${ }^{22}$. For CheckV we used the flag '--max_aai 95' to exclude closely related genomes in the CheckV database. For VIBRANT, we used the flag '--virome' to increase sensitivity. For viralComplete, completeness was determined based on the ratio of contig length to that of the corresponding genome from NCBI RefSeq. Completeness estimates $>100 \%$ were set to $100 \%$. Additionally, we benchmarked CheckV using genome fragments derived from NCBI Genbank genomes and used the flag '--max_aai 95' to exclude closely related genomes in the CheckV database.

Benchmarking detection of host regions on proviruses. To benchmark CheckV's detection of host regions, we constructed a mock dataset of proviruses: 382 viral genomes were downloaded from NCBI GenBank (after 1 June 2019) and paired with 76 GTDB genomes ( 71 bacterial, 5 archaeal). None of the 382 genomes were used to train CheckV (that is, selection of HMMs and bit-score thresholds). The pairing was performed at the genus level based on the annotated names of virus and host (for example, Escherichia phage paired with Escherichia bacterial genome). When multiple GTDB genomes were available for a given bacterial genus, we chose that with the highest CheckM quality score and selected a maximum of ten GenBank genomes per bacterial genus to reduce the influence of a few over-represented groups. Any GenBank or GTDB genome that was used at any stage for training CheckV was excluded. Proviruses were simulated at varying contig lengths $(5,10,20,50$ and $100 \mathrm{~kb})$ with varying levels of host contamination $(10,20$ and $50 \%$; defined as the percentage of contig length derived from the microbial genome). Microbial genome fragments were appended to either the $5^{\prime}$ or $3^{\prime}$ end of the viral fragment at random. As a negative control, we also simulated contigs that were entirely viral (that is, no flanking microbial region) at the same contig lengths.

Mock proviruses were used as input to CheckV using default parameters. For comparison, we also ran VIBRANT v.1.2.0 (ref. ${ }^{11}$ ), VirSorter v.1.0.5 (ref. ${ }^{10}$ ), PhiSpy v.3.7.8 (ref. ${ }^{23}$ ) and Phigaro v.2.2.5 (ref. ${ }^{24}$ ). All tools were run with default options with the exception of VIBRANT and VirSorter, which were run with the flag '--virome' to increase sensitivity. Nucleotide sequences were used as input to all tools, except PhiSpy, for which we first ran Prokka v.1.14.5 (ref. ${ }^{60}$ ) to generate the required input file. A contig was classified as a provirus if it contained a predicted viral region covering $<95 \%$ of its length. Each prediction was then classified as a true positive (provirus classified as provirus), false positive (viral contig classified as provirus), true negative (viral contig not classified as provirus) or false negative (provirus classified as provirus). For the true positives, we also compared the true and predicted lengths of the host region.

Application of CheckV to diverse viral genome collections. We downloaded 735,106 contigs $>5 \mathrm{~kb}$ from IMG/VR 2.0 (ref. ${ }^{25}$ ), after exclusion of viral genomes from cultivated isolates and proviruses identified from microbial genomes. We also downloaded 488,131 contigs $>5 \mathrm{~kb}$ or circular from the GOV 2.0 dataset $^{6}$ (datacommons.cyverse.org/browse/iplant/home/shared/iVirus/GOV2.0). These were used as input to CheckV to estimate the completeness, identify host-virus boundaries and predict closed genomes. When running the completeness module, we excluded perfect matches (100\% AAI and $100 \% \mathrm{AF})$ to prevent any DTR contig from matching itself in the database (since IMG/VR 2.0 and GOV 2.0 were used as data sources to form the CheckV database). A Circos plot ${ }^{61}$ was used to link IMG/ VR contigs to their top matches in the CheckV database. Protein-coding genes were predicted from proviruses using Prodigal and compared to HMMs from KEGG Orthology (release 2 October 2019) ${ }^{45}$ using hmmsearch from the HMMER package v.3.1b2 $\left(\leq 1 \times 10^{-5}\right.$ and score $\left.\geq 30\right)$. Pfam domains with the keyword 'integrase' and 'recombinase' were also identified across all proviruses.

The largest DTR contig we identified from IMG/VR was further annotated to illustrate the type of virus and genome organization represented (IMG ID: 3300025697_Ga0208769_1000001). Coding sequence prediction and functional annotations were obtained from $\mathrm{IMG}^{35}$. Annotation for virus hallmark genes including a terminase large subunit (TerL) and major capsid protein were confirmed via HHPred v.3.2.0 (ref. ${ }^{62}$ ) (databases included PDB 70_8, SCOPe70 2.07, Pfam-A 32.0 and CDD 3.18, score $>98$ ). A circular genome map was drawn with CGView ${ }^{63}$. To place this contig in an evolutionary context, we built a TerL phylogeny including the most closely related sequences from a global search for large phages ${ }^{42}$. The TerL amino acid sequence from the DTR contig was compared to all TerL sequences from the 'huge phage' dataset via blastp $\left(\leq 1 \times 10^{-5}\right.$, score $\geq 50$ ) to identify the 30 most similar sequences (sorted based on blastp bit-score) These reference sequences and DTR contigs were aligned with MAFFT v.7.407 (ref. ${ }^{64}$ ) using default parameters, the alignment automatically cleaned with trimAL v.1.4.rev15 with the option '--gappyout' ${ }^{\prime 65}$ and a phylogeny built with IQ-Tree v.1.5.5, with default model selection (optimal model suggested: LG+R4) ${ }^{66}$. The resulting tree was visualized with $\mathrm{iToL}^{67}$

Reporting Summary. Further information on research design is available in the Nature Research Reporting Summary linked to this article.

\section{Data availability}

The complete CheckV database, including HMMs, GenBank genomes and DTR contigs, is available at https://portal.nersc.gov/CheckV/.

\section{Code availability}

CheckV is written in Python and is freely available as open source software at https://bitbucket.org/berkeleylab/CheckV under a BSD license.

\section{References}

49. Goodacre, N. et al. A reference viral database (RVDB) to enhance bioinformatics analysis of high-throughput sequencing for novel virus detection. mSphere 3, e00069-18 (2018). 
50. El-Gebali, S. et al. The Pfam protein families database in 2019. Nucleic Acids Res. 47, D427-D432 (2019).

51. Finn, R. D. et al. The Pfam protein families database. Nucleic Acids Res. 38, D211-D222 (2010).

52. Haft, D. H. et al. TIGRFAMs and genome properties in 2013. Nucleic Acids Res. 41, D387-D395 (2013).

53. Eddy, S. R. Accelerated profile HMM searches. PLoS Comput. Biol. 7, e1002195 (2011)

54. Hyatt, D. et al. Gene and translation initiation site prediction in metagenomic sequences. Bioinformatics 28, 2223-2230 (2012).

55. Buchfink, B., Xie, C. \& Huson, D. H. Fast and sensitive protein alignment using DIAMOND. Nat. Methods 12, 59-60 (2015).

56. Camacho, C. et al. BLAST+: architecture and applications. BMC Bioinformatics 10, 421 (2009).

57. Jorgensen, T. S. et al. Hundreds of circular novel plasmids and DNA elements identified in a rat cecum metamobilome. PLoS ONE 9, e87924 (2014).

58. Martini, M. C. et al. Genomics of high molecular weight plasmids isolated from an on-farm biopurification system. Sci. Rep. 6, 28284 (2016).

59. Jorgensen, T. S. et al. Plasmids, viruses, and other circular elements in rat gut. Preprint at bioRxiv https://doi.org/10.1101/143420 (2017).

60. Seemann, T. Prokka: rapid prokaryotic genome annotation. Bioinformatics 30, 2068-2069 (2014).

61. Krzywinski, M. et al. Circos: an information aesthetic for comparative genomics. Genome Res. 19, 1639-1645 (2009).

62. Soding, J., Biegert, A. \& Lupas, A. N. The HHpred interactive server for protein homology detection and structure prediction. Nucleic Acids Res. 33, W244-W248 (2005).

63. Stothard, P. \& Wishart, D. S. Circular genome visualization and exploration using CGView. Bioinformatics 21, 537-539 (2005).

64. Katoh, K. \& Standley, D. M. MAFFT multiple sequence alignment software version 7: improvements in performance and usability. Mol. Biol. Evol. 30, 772-780 (2013).

65. Capella-Gutierrez, S., Silla-Martinez, J. M. \& Gabaldon, T. trimAl: a tool for automated alignment trimming in large-scale phylogenetic analyses. Bioinformatics 25, 1972-1973 (2009).
66. Nguyen, L. T. et al. IQ-TREE: a fast and effective stochastic algorithm for estimating maximum-likelihood phylogenies. Mol. Biol. Evol. 32, 268-274 (2015)

67. Letunic, I. \& Bork, P. Interactive Tree Of Life (iTOL) v4: recent updates and new developments. Nucleic Acids Res. 47, W256-W259 (2019).

\section{Acknowledgements}

We thank R. Cavicchioli for providing additional information on the sample from which the largest DTR contig was assembled. The work conducted by the US Department of Energy Joint Genome Institute is supported by the Office of Science of the US Department of Energy under contract no. DE-AC02-05CH11231. This work was also supported by grant no. 2016/23218-0 from the São Paulo Research Foundation (FAPESP). A.P.C. received a scholarship (no. 2018/04240-0) from FAPESP.

\section{Author contributions}

S.N. and S.R conceived the project. S.N. drafted the manuscript, developed algorithms, databases and software, performed benchmarking and analyzed IMG/VR genome quality statistics. S.R. provided feedback, performed troubleshooting, analyzed the largest DTR contig from IMG/VR, analyzed IMG/VR genome quality statistics and contributed to manuscript writing. A.P.C. provided feedback, improved the code base and packaged the software. F.S. performed the analysis of GVMAGs. N.C.K. supervised the project. All authors reviewed and approved the manuscript.

\section{Competing interests}

The authors declare no competing interests.

\section{Additional information}

Extended data is available for this paper at https://doi.org/10.1038/s41587-020-00774-7.

Supplementary information is available for this paper at https://doi.org/10.1038/ s41587-020-00774-7.

Correspondence and requests for materials should be addressed to S.N. or N.C.K. Reprints and permissions information is available at www.nature.com/reprints. 
A
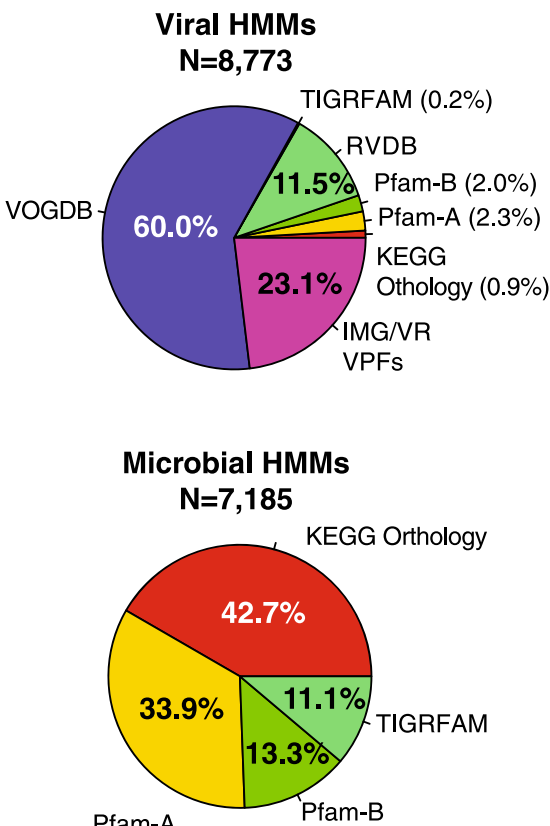

Pfam-A
Pfam-B
B

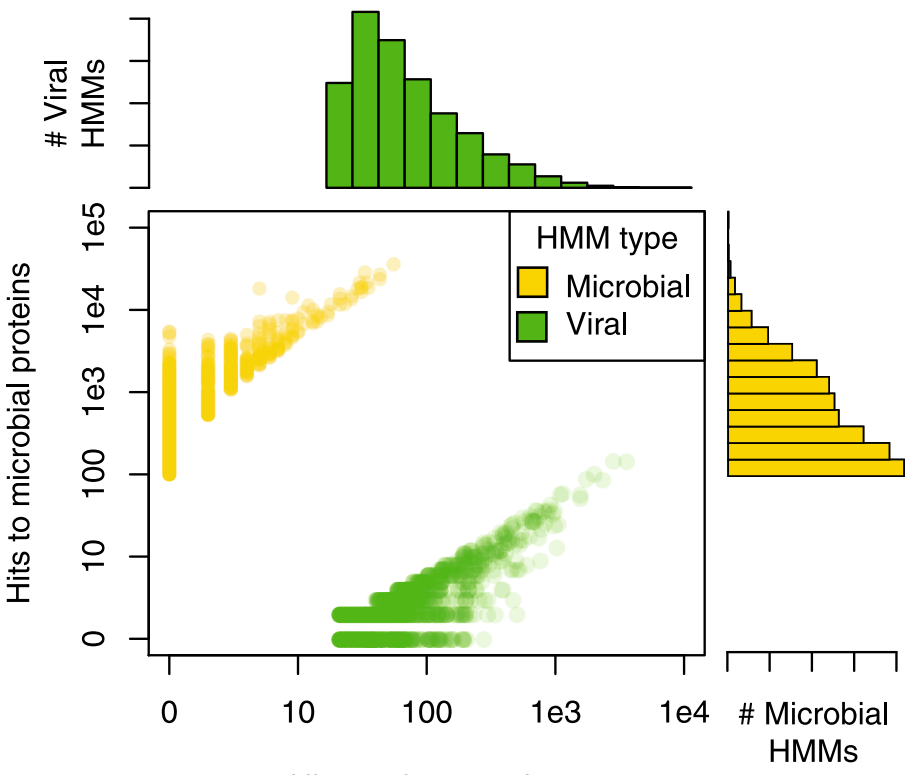

Hits to viral proteins

Extended Data Fig. 1 | CheckV database of viral- and microbial-specific HMMs. a) Non-redundant viral and microbial HMMs were selected from seven reference databases. $\mathbf{b})$ The distribution of the number of hits to viral and microbial proteins for the selected HMMs shown in panel A. 
A
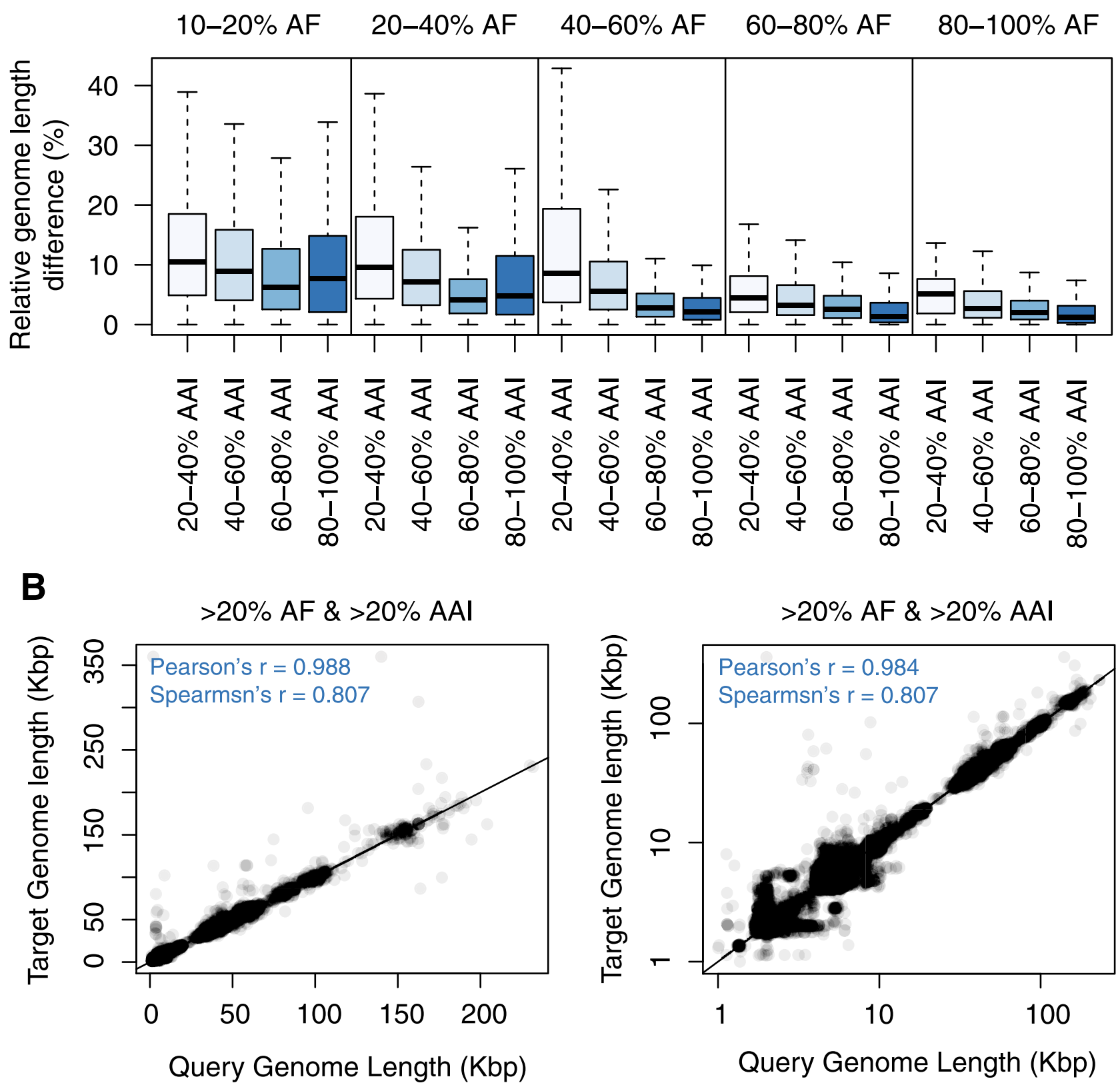

Extended Data Fig. 2 | Variation in genome size between viruses. The relatedness between all CheckV reference genomes was estimated based on their average amino acid identity (AAI) and alignment fraction (AF). For box plots, the middle line denotes the median, the box denotes the interquartile range $(I Q R)$, and the whiskers denote $1.5 \times$ the IQR. a) The relative difference in genome length for viruses with varying degrees of relatedness. b) Scatterplots showing genome sizes between related viruses. The right panel shows genome sizes on a log10 scale. 
A

Publicly-available metagenomic assemblies IMG/M $\left(6.6 \times 10^{12} \mathrm{bp}\right)$

MGnify $\left(1.1 \times 10^{12} \mathrm{bp}\right)$

Pasolli et al. $2019\left(1.4 \times 10^{12} \mathrm{bp}\right)$

Nayfach et al. $2019\left(6.0 \times 10^{11} \mathrm{bp}\right)$

Soto Perez \& Bisanz et al. $2019\left(1.9 \times 10^{10} \mathrm{bp}\right)$

Gregory et al. $2019\left(1.9 \times 10^{10} \mathrm{bp}\right)$

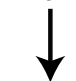

Metagenomic contigs

$\mathrm{N}=14.4$ billion $\left(9.7 \times 10^{12} \mathrm{bp}\right)$

- Direct terminal repeat (DTR) $\geq 20 \mathrm{bp}$

- Contig length $\geq 2 \mathrm{Kbp}$

DTR contigs

$\mathrm{N}=751,567$

- Remove low complexity DTRs

- Remove highly repetetive DTRs

- Remove duplicated contigs

Filtered DTR contigs

$\mathrm{N}=686,030$

$\downarrow \begin{aligned} & \text { - CheckV marker genes } \\ & \text { - VirFinder }\end{aligned}$

DTR viral contigs

$\mathrm{N}=116,666$

-Validate completeness using CheckV and taxonomic marker genes

Validated DTR viral contigs $\mathrm{N}=76,262$

$$
\downarrow \begin{aligned}
& \text { - Centroid clustering } \\
& -\geq 95 \% \text { ANI } \\
& -\geq 85 \% \text { AF of both contigs }
\end{aligned}
$$

Non-redundant DTR viral contigs $\mathrm{N}=39,117$
B
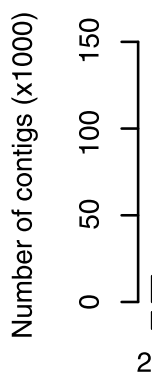

D

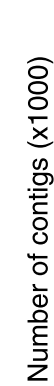

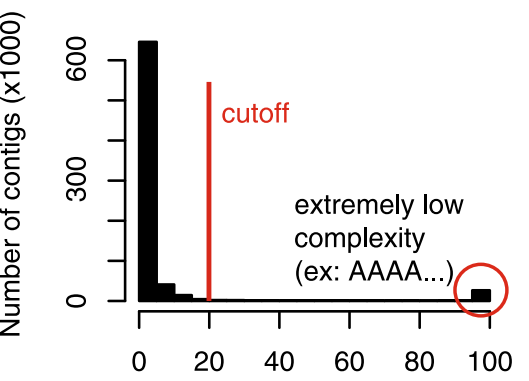

Low complexity (\% of repeat length)
C

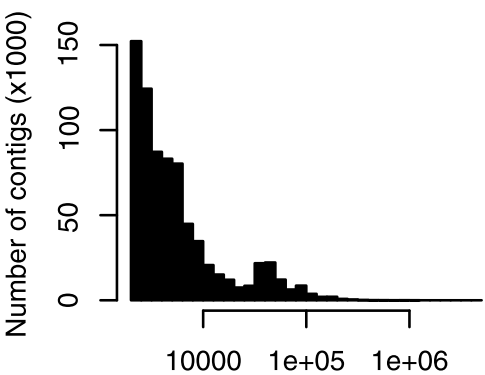

E

DTR contig length (bp)

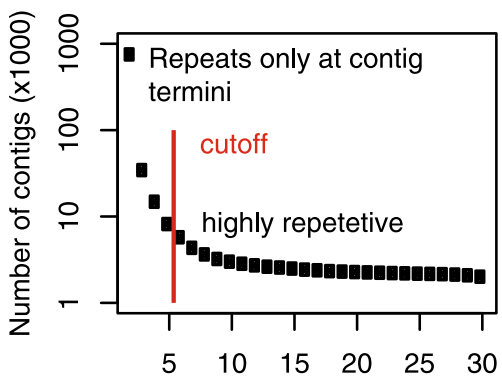

Repeat frequency (min. \# per contig)
$\mathbf{F}$

CheckV Marker Genes viral genes $>$ microbial genes viral genes $>$ plasmid genes

VirFinder

p-value $<0.001$ plasmid genes $=0$ microbial genes $<2$
DTR Viral Contigs
$\mathrm{N}=116,666$

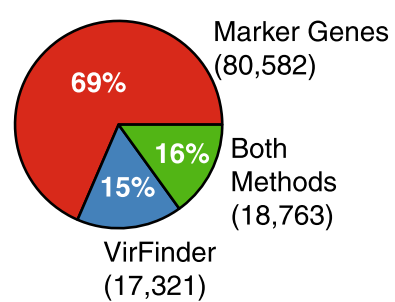

$(17,321)$

Extended Data Fig. 3 | Identification of DTR viral contigs. a) Publicly available metagenomes were systematically mined for 76,262 DTR viral contigs, resulting in 39,117 non-redundant contigs after de-replication at 95\% ANI over $85 \%$ the length of both sequences. b-e) Summary statistics across the 751,567 DTR contigs before filtering. b) Distribution of the length of direct terminal repeats (DTRs). A considerable number of DTRs occur at specific lengths (for example 55, 77, $99 \mathrm{bp}$ ). These odd-numbered lengths likely correspond with k-mer lengths utilized by various metagenomic assembly tools. When faced with assembling reads from a circular template, they appear to break the contig in a random location and leave behind a repeated sequence at the start and end of the contig equal to the k-mer length. c) The length (log scale) of all DTR contigs. d) A small number of contigs are likely false positives due to a low complexity repeat (for example AAAAAA...) or e) a highly repetitive repeat (that is occurring not just at termini). f) After removing potentially spurious complete genomes, the DTR contigs were screened for viral signatures, revealing 116,666 viral contigs. These were identified using a combination of CheckV's marker genes, plasmid genes from recent publications, and VirFinder. 
A
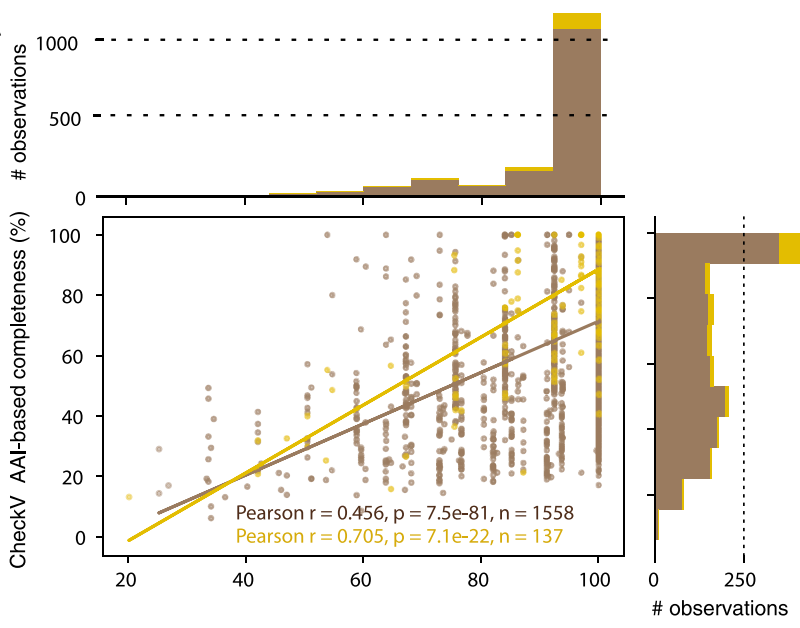

NCVOG completeness $(\%)$

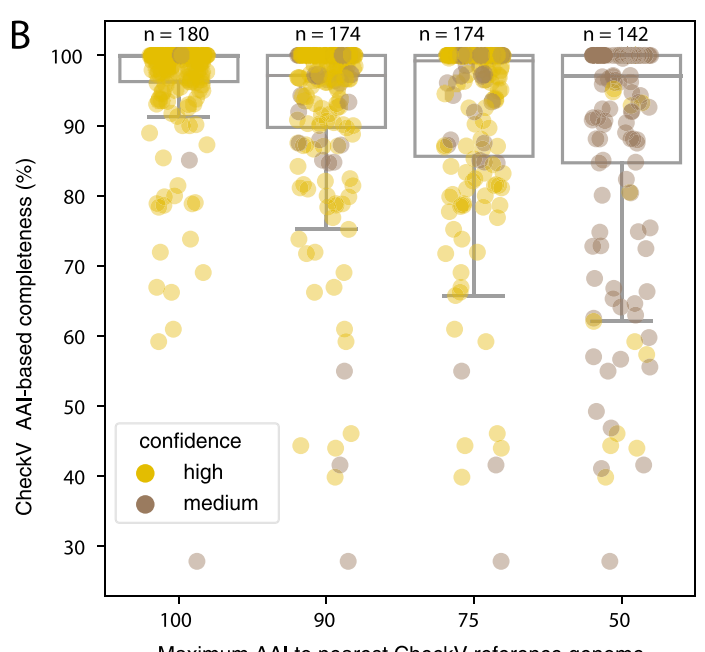

Maximum AAI to nearest CheckV reference genome

Extended Data Fig. 4 | Evaluation of CheckV completeness estimates for giant virus metagenome assembled genomes (GVMAGs) and nucleocytoplasmic large DNA virus (NCLDV) isolate genomes. a) Correlation between CheckV completeness (medium and high confidence AAI-based estimates) versus low-copy number NCVOG completeness estimates for 2,074 GVMAGs. Points in the scatter plot indicate GVMAGs that had a high (yellow) or medium (brown) confidence CheckV completeness estimate. Histograms indicate the total number of GVMAGs across different completeness intervals for both approaches. GVMAGs with undetermined or low-confidence CheckV estimates were excluded from the analysis $(n=379)$. Completeness estimates above $100 \%$ were set to $100 \%$. P values were calculated using a two-sided Pearson regression. $\mathbf{b}) \mathrm{CheckV}$ completeness estimates for 182 NCLDV isolate genomes after excluding closely related CheckV reference genomes. The maximum amino acid identity between CheckV references and NCLDV isolate genomes is indicated by the plot labels. Center lines of box plots represent the median, bounds of boxes the lower and upper quartile, whiskers extend to points that lie within 1.5 interquartile range of the lower and upper quartile. Each data point represents the completeness estimate for a NCLDV reference genome, where the color indicates the CheckV confidence level. Completeness estimates above $100 \%$ were set to $100 \%$. 
A

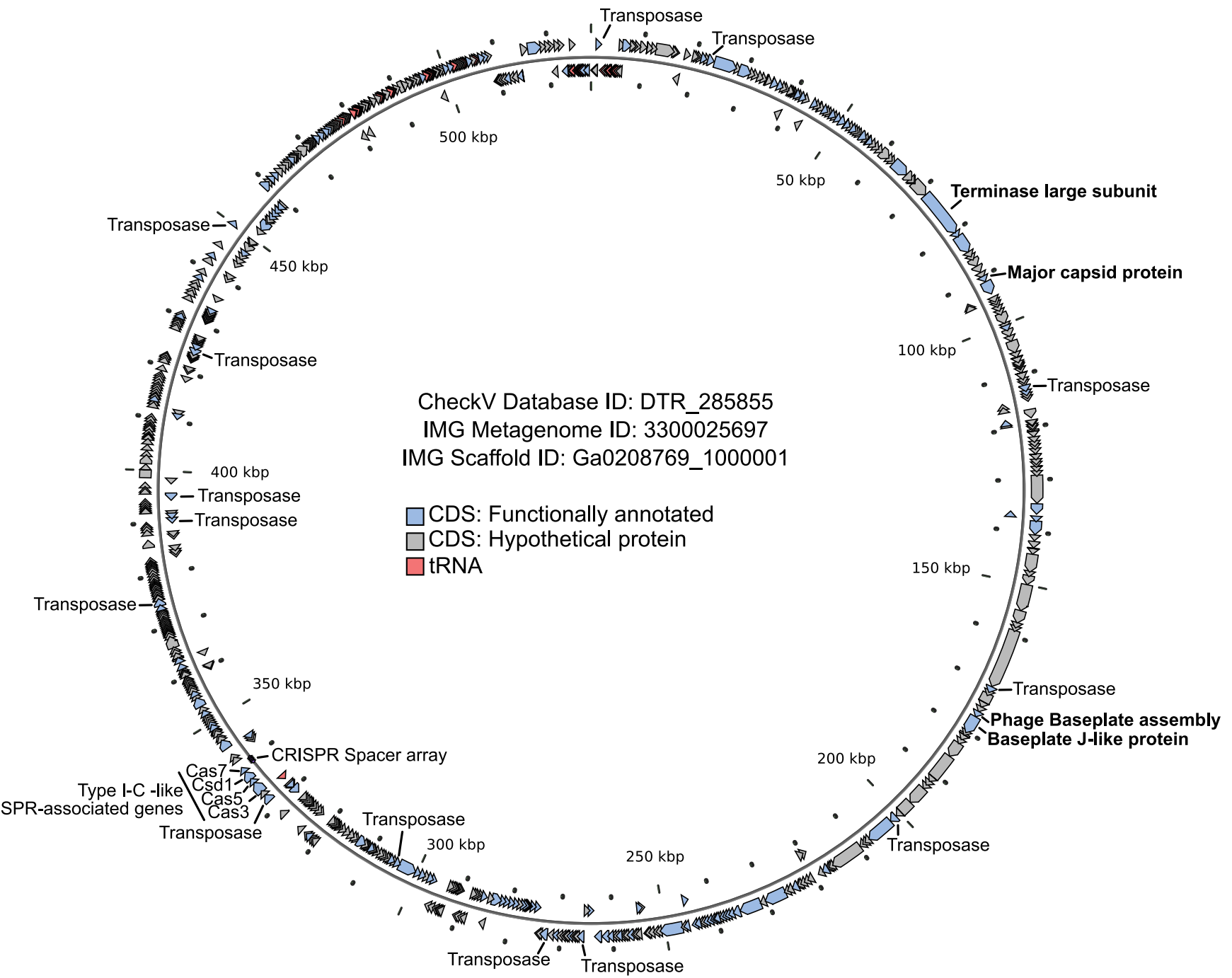

B

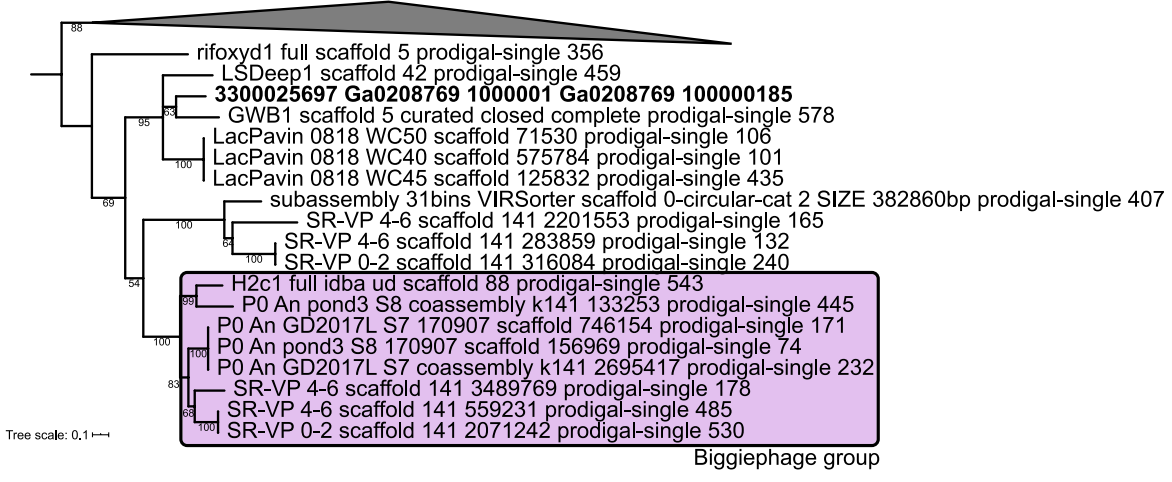

Groundwater, Rifle, Colorado

Landsort Deep, Baltic Sea

Ace Lake, Antarctica

Groundwater, Rifle, Colorado

Lake Pavin, France

Lake Pavin, France

Lake Pavin, France

East River, Colorado, USA

Vernal pool mud, Lake County, California Vernal pool mud, Lake County, California Vernal pool mud, Lake County, California Soil, East River, Colorado

Sewerage pond, Modesto, California

Groundwater, Modesto, California

Groundwater, Modesto, California

Vernal pool mud, Lake County, California

Vernal pool mud, Lake County, California Vernal pool mud, Lake County, California

Extended Data Fig. 5 | Genome map and phylogeny of the largest complete genome from IMG/VR. a) Genome map of 528,258 bp putative circular metagenomic contig (IMG identifier: Ga0222679_1000001), sampled from Ace Lake in Antarctica. Annotations were obtained from IMG and manual annotation of phage proteins (terminase and major capsid protein) via HHPred. b) TerL phylogeny of the circular metagenomic contig with the most closely related sequences from a global search for large phages. 

TAGCAA... Tि

Prophages

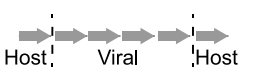

ITRs (>20-bp) TAGCAA.. ..TTGCTA
B
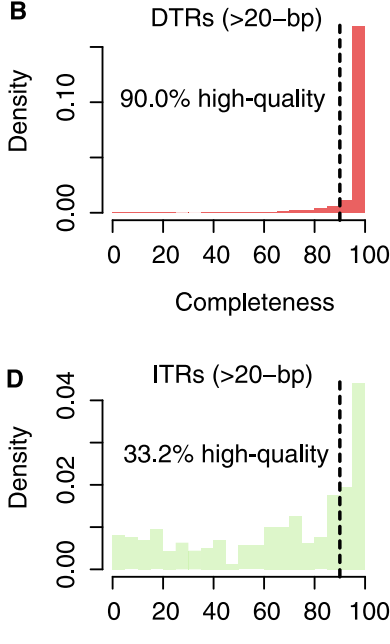

C

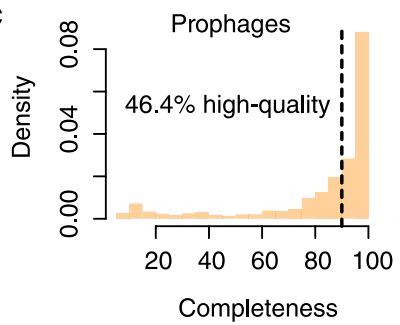

E

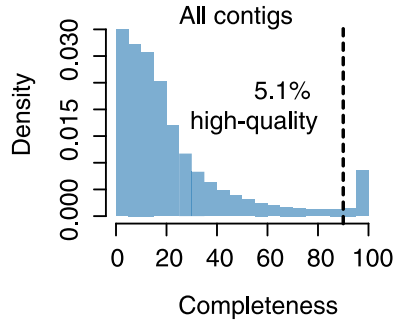

Extended Data Fig. 6 | Evaluating metagenomic signatures of complete viral genomes. Putative complete viral genomes were identified from the IMG/ VR database based on either A) DTRs $(N=15,211), B)$ proviruses with flanking host regions $(N=451)$, or $C)$ ITRs $(N=624)$. In parallel, the completeness of IMG/VR contigs was estimated using the AAI-based approach, using only high- and medium-confidence level estimates. The histograms above show the distribution of completeness for each signature of complete genomes (high- and medium-confidence estimates only), as well as the distribution of completeness across all other IMG/VR contigs. The text indicates the percent of IMG/VR contigs in each category that are classified as high-quality (that is $>90 \%$ estimated completeness). 


\section{IMG/VR contigs}

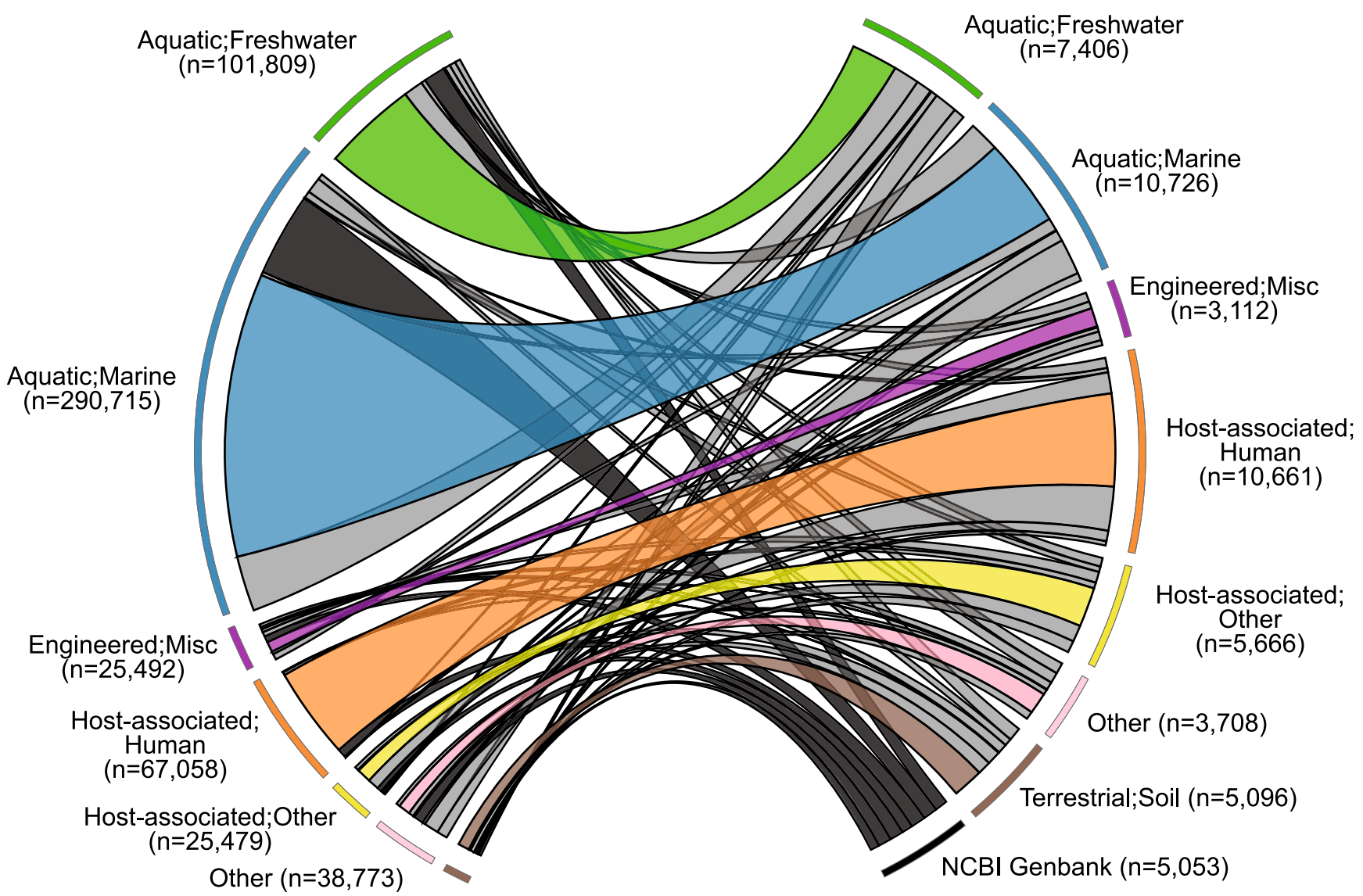

Terrestrial;Soil $(n=16,124)$

Extended Data Fig. 7| Association between IMG/VR contigs and CheckV reference genomes. IMG/VR contigs (left) are classified by the biome of their original metagenomes and connected to their top hit in the CheckV database based on amino acid identity (right). Cases in which IMG/VR contigs and CheckV references are derived from the same habitat (for example marine IMG/VR contig and marine CheckV reference) are colored by biome, while other cases are colored in grey. 
A

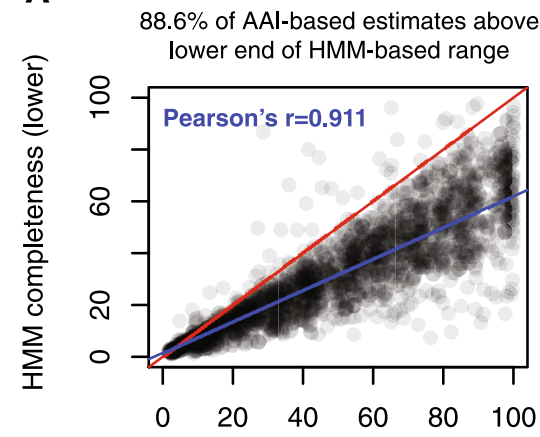

AAl completeness
B

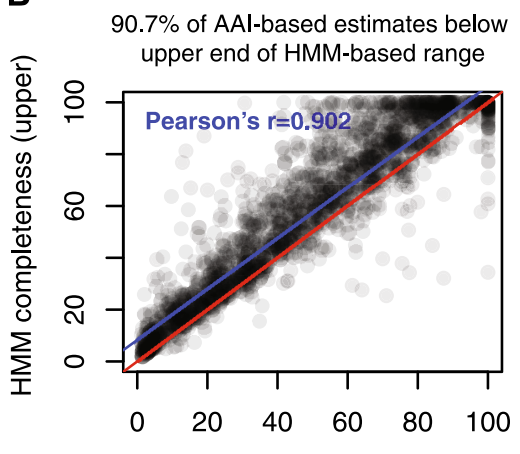

AAI completeness
C

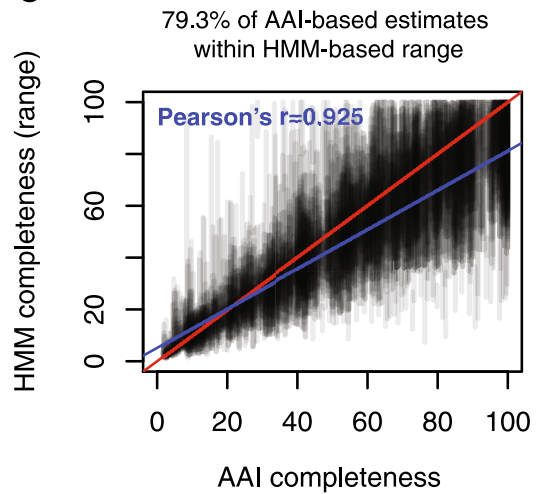

Extended Data Fig. 8 | Comparison between AAI and HMM-based completeness estimates. The analysis above was based on 568,096 IMG/VR contigs with both AAI- and HMM-based completeness estimates. Note that the AAl-based approach results in a point estimate whereas the HMM-based approach results in a range representing the estimated $90 \%$ confidence interval based on the empirical distribution of reference genome lengths sharing the same HMMs. A random subset of points shown for each plot. a) Comparison between AAI-based estimates and the lower end of the HMM-based range. As expected, most AAI-based estimates fall above the lower end of the HMM-based range. This percentage is lower than 95\% (the target value) likely due to error in AAI- and HMM-based estimates. Each point is a single contig. b) Comparison between AAI-based estimates and the upper end of the HMM-based range. Each point is a single contig. c) Comparison between AAI-based estimates and the midpoint of the HMM-based range. Each line is a single contig. For all figures, the line of identity is indicated in red while the line of best fit is indicated in blue. The Pearson correlation coefficient is shown in blue. 
A

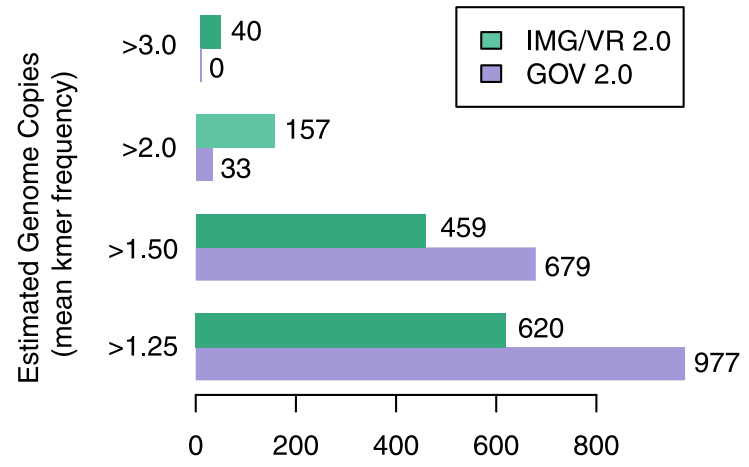

Number of viral contigs
B

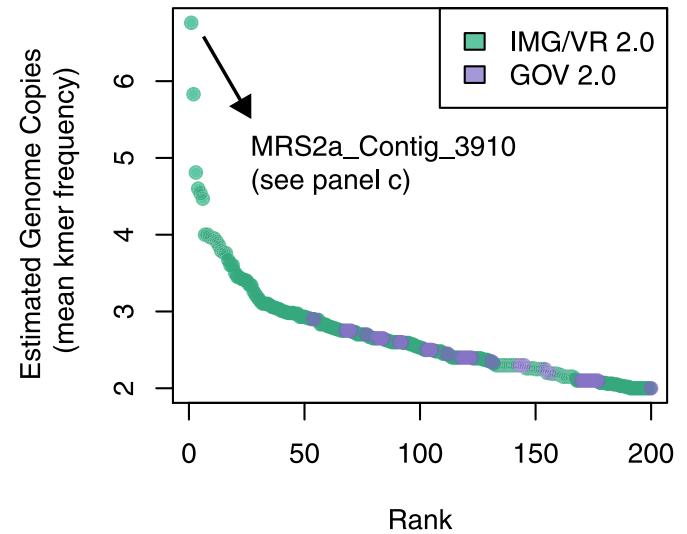

C IMG taxon id: 2124908027

Metagenome: Miscanthus rhizosphere microbial communities from Kellogg Biological Station Scaffold: MRS2a_Contig_3910; Length: 279,201 bp; GC content: 59\%

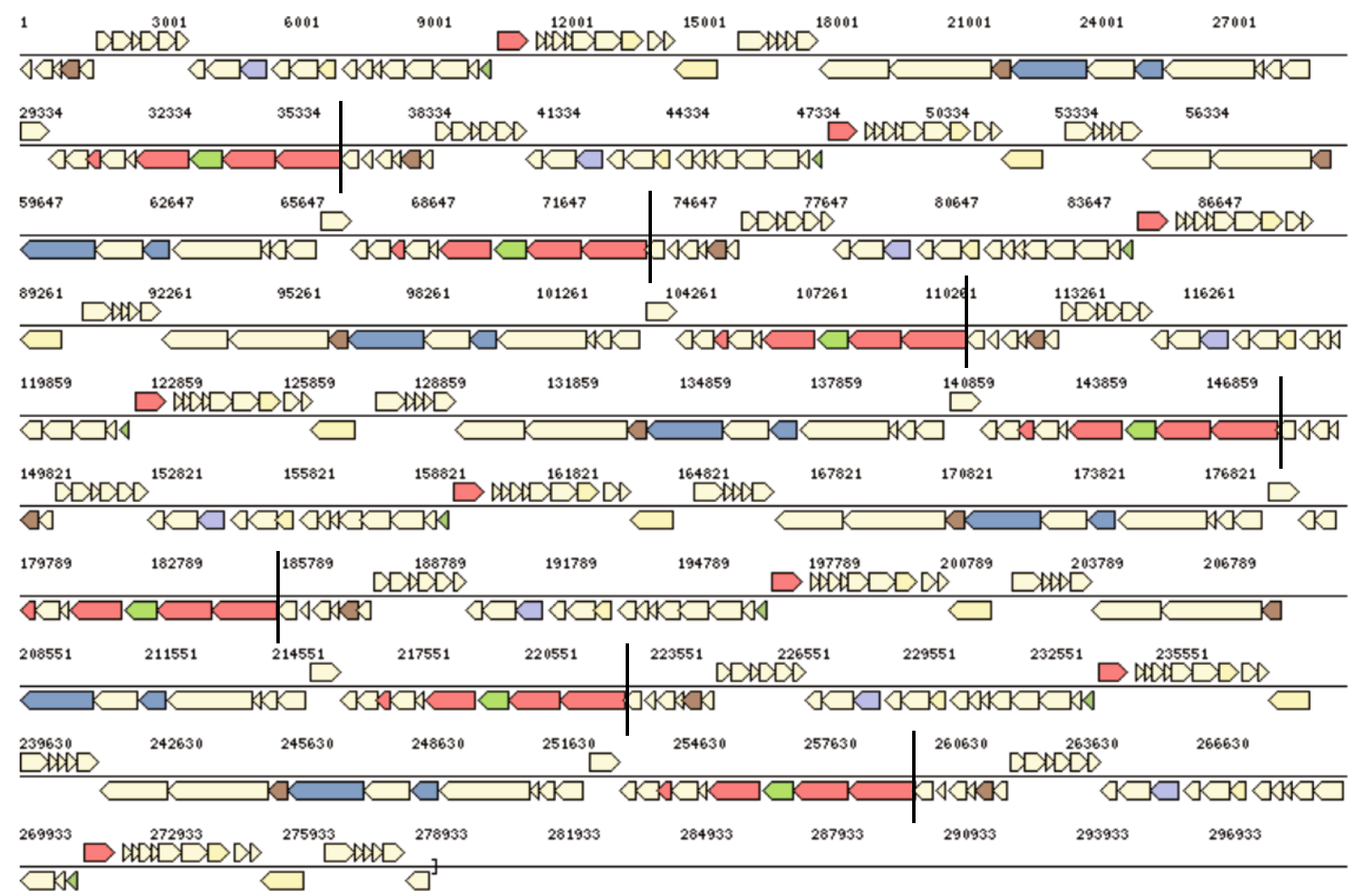

Extended Data Fig. 9 | Identification of contigs with concatenated genomes in public databases. CheckV was used to quantify the average $k$-mer frequency for viral contigs in IMG/VR and the GOV. A contig that contains exactly one genome copy should have a $k$-mer frequency close to 1.0 (all kmers are unique). Contigs that contain the same repeated genome (for example concatemers) will have $k$-mer frequencies above 1.0. a) The number of viral contigs at different cutoff points for the $k$-mer frequency statistic. b) Top 100 contigs with the highest $k$-mer frequencies. c) An example of a $279,201 \mathrm{bp}$ contig in IMG/VR with the same sequence repeated more than $7 x$. 
A

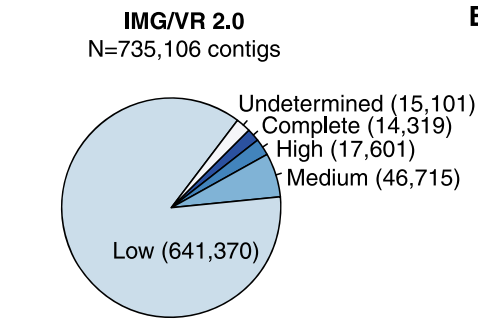

AAl-based

( $\mathrm{N}=589,654)$

$\%$ of predictions

D

IMG/VR 2.0

$\mathrm{N}=735,106$ contigs

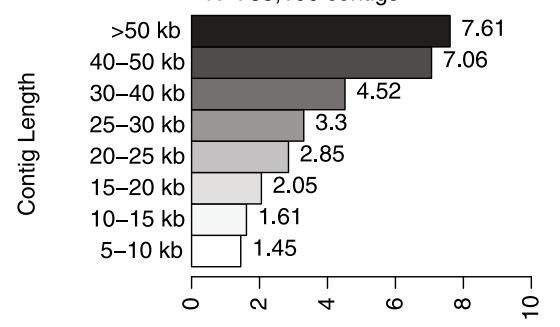

$\%$ of contigs classified as provirus
B

Global Ocean Virome (GOV 2.0)
$\mathrm{N}=488,130$ contigs

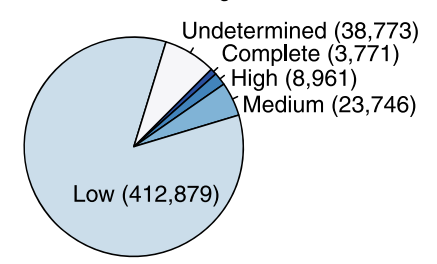

AAl-based

$(\mathrm{N}=375,038)$

HMM-based $(\mathrm{N}=74,319)$

$\%$ of predictions

E

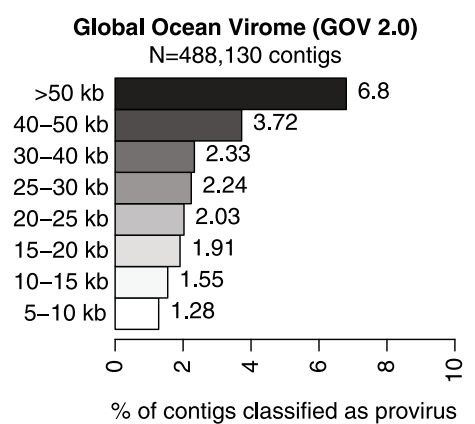

C

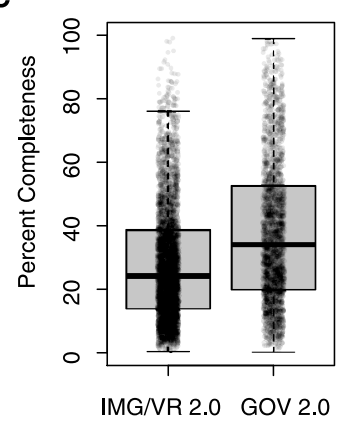

$\mathbf{F}$

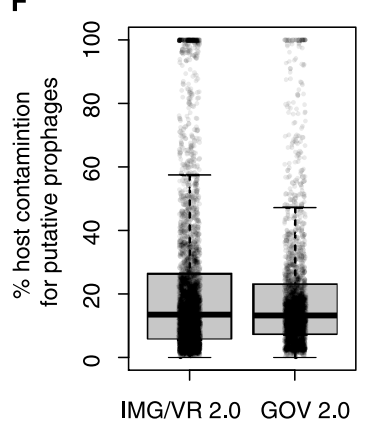

Extended Data Fig. 10 | Application of CheckV to the IMG/VR 2.0 and the Global Ocean Virome 2.0 datasets. a) Quality tiers across viral contigs from IMG/VR 2.0 and b) the GOV 2.0 dataset. The bar plots indicate the \% of completeness estimates made with the AAI- or HMM-based approaches. c) Distribution of AAI-based completeness across contigs from each dataset. d) Percent of contigs classified as a provirus for IMG/VR 2.0 and $\mathbf{e}$ ) for GOV 2.0. f) Distribution of host contamination (that is percent of length derived from host regions on proviruses) across datasets. For box plots, the middle line denotes the median, the box denotes the interquartile range (IQR), and the whiskers denote $1.5 \times$ the IQR. 


\section{Reporting Summary}

Nature Research wishes to improve the reproducibility of the work that we publish. This form provides structure for consistency and transparency in reporting. For further information on Nature Research policies, see our Editorial Policies and the Editorial Policy Checklist.

\section{Statistics}

For all statistical analyses, confirm that the following items are present in the figure legend, table legend, main text, or Methods section.

n/a Confirmed

$\bigotimes$ The exact sample size $(n)$ for each experimental group/condition, given as a discrete number and unit of measurement

$\bigotimes$ A statement on whether measurements were taken from distinct samples or whether the same sample was measured repeatedly

The statistical test(s) used AND whether they are one- or two-sided

Only common tests should be described solely by name; describe more complex techniques in the Methods section.

$\bigotimes$ A description of all covariates tested

$\bigotimes$ A description of any assumptions or corrections, such as tests of normality and adjustment for multiple comparisons

A full description of the statistical parameters including central tendency (e.g. means) or other basic estimates (e.g. regression coefficient)

AND variation (e.g. standard deviation) or associated estimates of uncertainty (e.g. confidence intervals)

For null hypothesis testing, the test statistic (e.g. $F, t, r$ ) with confidence intervals, effect sizes, degrees of freedom and $P$ value noted

Give $P$ values as exact values whenever suitable.

Х $\square$ For Bayesian analysis, information on the choice of priors and Markov chain Monte Carlo settings

Х $\square$ For hierarchical and complex designs, identification of the appropriate level for tests and full reporting of outcomes

$\square \bigotimes$ Estimates of effect sizes (e.g. Cohen's $d$, Pearson's $r$ ), indicating how they were calculated

Our web collection on statistics for biologists contains articles on many of the points above.

\section{Software and code}

Policy information about availability of computer code

Data collection No software was used

Data analysis HMMER v3.1b2, VIBRANT v1.2.0, VirSorter v.1.0.5, PhiSpy v.3.7.8, 'all_pairs' function in the SetSimilaritySearch Python package (https:// github.com/ekzhu/SetSimilaritySearch), Prodigal v2.6.3, DIAMOND v0.9.30, BLAST+ v2.9.0, VirFinder v1.1, Phigaro v0.1.5.0, Prokka v1.14.5, HHPred v3.2.0, CGView, MAFFT v7.407, trimAL v1.4.rev15, IQ-Tree v1.5.5, iToL, viralComplete

For manuscripts utilizing custom algorithms or software that are central to the research but not yet described in published literature, software must be made available to editors and

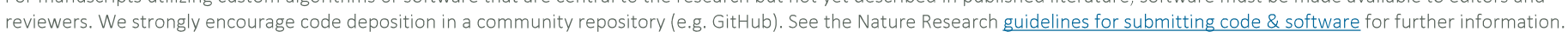

\section{Data}

Policy information about availability of data

All manuscripts must include a data availability statement. This statement should provide the following information, where applicable:

- Accession codes, unique identifiers, or web links for publicly available datasets

- A list of figures that have associated raw data

- A description of any restrictions on data availability

The complete CheckV database, including HMMs, GenBank genomes, and DTR contigs are available at https://portal.nersc.gov/CheckV/. Quality statistics and listing of complete viral genomes from IMG/VR will be available in the next version scheduled for release on September 2020. 
Please select the one below that is the best fit for your research. If you are not sure, read the appropriate sections before making your selection. \ Life sciences Behavioural \& social sciences Ecological, evolutionary \& environmental sciences

\section{Life sciences study design}

All studies must disclose on these points even when the disclosure is negative.

Sample size n/a
Data exclusions n/a
Replication n/a
Randomization $n$ n/a

\section{Reporting for specific materials, systems and methods}

We require information from authors about some types of materials, experimental systems and methods used in many studies. Here, indicate whether each material, system or method listed is relevant to your study. If you are not sure if a list item applies to your research, read the appropriate section before selecting a response.

\begin{tabular}{|c|c|c|c|}
\hline \multicolumn{2}{|r|}{ Materials \& experimental systems } & \multicolumn{2}{|c|}{ Methods } \\
\hline$n / a$ & Involved in the study & $n / a$ & Involved in the study \\
\hline Х & $\square$ Antibodies & Х & $\square$ ChIP-seq \\
\hline Х & $\square$ Eukaryotic cell lines & $\bigotimes$ & Flow cytometry \\
\hline 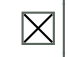 & Palaeontology and archaeology & Х & $\square$ MRI-based neuroimaging \\
\hline 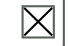 & $\square$ Animals and other organisms & & \\
\hline Х & $\square$ Human research participants & & \\
\hline Х & $\square$ Clinical data & & \\
\hline$\bigotimes$ & $\square$ Dual use research of concern & & \\
\hline
\end{tabular}

